\title{
Finite quantum gravity in dS and AdS spacetimes
}

\author{
Alexey S. Koshelev ${ }^{\dagger}$ \\ Departamento de Física, Centro de Matemática e Aplicações (CMA-UBI), \\ Universidade da Beira Interior, 6200 Covilhã, Portugal \\ and Theoretische Natuurkunde, Vrije Universiteit Brussel and The International Solvay Institutes, \\ Pleinlaan 2, B-1050 Brussels, Belgium \\ K. Sravan Kumar \\ Departamento de Física, Centro de Matemática e Aplicações (CMA-UBI), \\ Universidade da Beira Interior, 6200 Covilhã, Portugal \\ and Department of Physics, Southern University of Science and Technology, \\ Shenzhen 518055, China \\ Leonardo Modesto \\ Department of Physics, Southern University of Science and Technology, Shenzhen 518055, China \\ Lesław Rachwal ${ }^{\S}$ \\ Instituto de Física, Universidade de Brasília, 70910-900 Brasília, DF, Brazil
}

(Received 20 April 2018; published 10 August 2018)

\begin{abstract}
We hereby study the properties of a large class of weakly nonlocal gravitational theories around the (anti-) de Sitter spacetime background. In particular, we explicitly prove that the kinetic operator for the graviton field has the same structure as the one in Einstein-Hilbert theory around any maximally symmetric spacetime. Therefore, the perturbative spectrum is the same as standard general relativity, while the propagator on any maximally symmetric spacetime is a mere generalization of the one from Einstein's gravity derived and extensively studied in several previous papers. At quantum level the range of theories presented here is superrenormalizable or finite when proper (not affecting the propagator) terms cubic or higher in curvatures are added. Finally, it is proven that for a large class of nonlocal theories, which in their actions do involve neither the Weyl nor the Riemann tensor, the theory is classically equivalent to the Einstein-Hilbert one with a cosmological constant by means of a metric field redefinition at any perturbative order.
\end{abstract}

DOI: $10.1103 /$ PhysRevD.98.046007

\section{INTRODUCTION}

In previous studies it has been extensively shown that a class of weakly nonlocal theories of gravity is unitary (ghost-free) and perturbatively superrenormalizable or finite in the framework of quantum field theory [1-11]. These works mostly concentrated on the perturbative theory around the flat Minkowski spacetime. The very foundations of the theory are the following: (i) general covariance;

\footnotetext{
*Corresponding author.

lmodesto@sustc.edu.cn

talexey@ubi.pt

*sravan@ubi.pt

§rzerach@gmail.com

Published by the American Physical Society under the terms of the Creative Commons Attribution 4.0 International license. Further distribution of this work must maintain attribution to the author(s) and the published article's title, journal citation, and DOI. Funded by SCOAP ${ }^{3}$.
}

(ii) weak nonlocality (or quasipolynomiality) [12]; (iii) unitarity (ghost freedom); and (iv) superrenormalizability or finiteness at quantum level. ${ }^{1}$ The new class of generally covariant theories differs from Einstein's gravity because of the weak nonlocality, which makes it possible to achieve unitarity and superrenormalizability at the same time, and at any order in the perturbative loop expansion. Nevertheless,

\footnotetext{
${ }^{1}$ We here would like to point out that in this paper all the results are proved in dimensional regularization scheme (DIMREG). This is to clarify the difference with the Wilsonian point of view and the functional renormalization group approach where the cutoff is taken seriously. We will use the standard DIMREG scheme adopted in QED, in QCD, and all the Standard Model of particle physics. However, our results are, of course, independent of the regularization scheme. We can, for example, use the cutoff regularization scheme with Pauli-Villars fields as it is done in QED, and we end up with exactly the same results found in DIMREG. Finally, the readers interested in the analysis of nonlocal theories carried out in the cutoff regularization scheme are referred to [13].
} 
the theory is not unique and all the freedom is mainly encoded in one, two, or three form factors (entire functions) with very specific asymptotic limits in the ultraviolet (UV) and infrared (IR) regimes in order to have a well defined quantum field theory.

We here study the same range of weakly nonlocal theories around maximally symmetric spacetimes (MSS) applying exactly the same logic so successfully implemented for theories around the Minkowski vacuum. In particular, we show that the kinetic operator for the gravitational fluctuations $\mathbf{h}$ resumes exactly the Einstein-Hilbert one up to some multiplicative factors. For this achievement, we explicitly show the results for the expansion of the action at the second order in $\mathbf{h}$ around a MSS. Therefore, all the results concerning the propagator on (anti-) de Sitter [(A)dS] spaces for the Einstein-Hilbert action can be exported and applied to the quasipolynomial theories, too. In particular, for one out of the two classes of theories, which we extensively study in this paper, we prove by the means of a field redefinition that at the perturbative level, but to all perturbative orders in the field redefinition, the nonlocal action is classically equivalent to the Einstein-Hilbert one in the presence of a cosmological constant. The proof is based on a field redefinition theorem that was already applied in [14] to the theory around the Minkowski vacuum.

There are several good theoretical as well as observational reasons to study the class of gravitational theories around MSS and not only around flat spacetime vacuum. Primarily, the true gravitational vacuum in quantum field theory is not precisely located as suggested by the cosmological constant problem. This has to do in other disguises with the gravitational effect of the zero modes of the simple quantized harmonic oscillator. (The last one works as a toy model for any perturbative quantum field theory (QFT), when it is treated as a theory of free propagating excitations.) Therefore, the flat Minkowski spacetime may not be the correct gravitational vacuum, and in some theories this state may even decay (via the spontaneous production of ghosts) as in higher derivative models of gravity. It may happen that the perturbative calculus around such false vacua is very fast divergent and not reliable due to the presence of different types of instabilities such as ghosts (negative norm states) or tachyons (negative mass states). A rescue could be to look for another vacuum state and to study quantum perturbations around the new vacuum. The MSS are the only other spacetimes where the number of local generators is not in conflict with the one of the Poincaré group for the flat spacetime. On MSS the spacetime symmetries are as rich as when on flat spacetime; hence MSS is potentially another good vacuum state. (We remind the reader that the vacuum state is a state of quite high symmetry.) On such a spacetime we do not violate homogeneity nor isotropy and the group of symmetries is only changed from $S O(1,3)$ into $S O(2,2)$ in the case of AdS in $D=4$ spacetime dimensions. In the case of dS the group of isometries remains the same. These new different vacua may be perturbatively unreachable from the original one; therefore, by studying quantum theories around (A)dS spacetimes we actually do nonperturbative physics from the flat spacetime perspective. Additionally, different backgrounds can be viewed as a resummation of collective gravitational fluctuations around an initial background.

On the other hand, the inclusion of background spacetimes of constant curvature is a very mild modification that can be treated exactly without tremendous efforts in computations. Therefore, it is an interesting laboratory to study perturbative implications of the same theory, but on different maximally symmetric backgrounds. For example, we can play with the value of the curvature radius of the background and we can easily check the claims about background independence of nonlocal theories. Since AdS spacetimes gained a lot of attention in the past two decades, mainly due to the AdS/CFT conjecture, it is also highly desirable to have a gravitational version of nonlocal theories formulated on general AdS backgrounds. This could be viewed as a first step toward the investigation of the gauge-gravity duality in a class of weakly nonlocal gravitational theories consistent at quantum level.

The cosmological constant $a_{\Lambda}$ appearing in the tree-level action should be understood as a new coupling constant of gravitational character, and not as a special matter source. Moreover, the cosmological constant term is an IR completion of the theory when we introduce all possible operators with a fixed number of derivatives. In the case of the cosmological constant we actually add a generally covariant term with no derivatives at all. And then the following issue arises that for consistency we should quantize physical theory around on-shell background, i.e., such that it solves exactly classical equations of motion. If we have the cosmological constant in the action, then the flat spacetime is not a solution anymore and we have to study the theory around Einstein spaces. The de Sitter and anti-de Sitter spacetimes serve as examples of such background spacetimes.

However, here we want to remark that it is also possible to pursue a different idea that the cosmological constant $\Lambda_{\mathrm{cc}}$ may not be present in the action. The background does not have to be on-shell with respect to the equations for the perturbations, and only at the end should the physical theory for the on-shell Minkowski background without $\Lambda_{\mathrm{cc}}$ be considered. If $\Lambda_{\mathrm{cc}}$ is in the action, then the fluctuations must be analyzed around on-shell (A)dS spacetimes. On the other hand, from the mathematical point of view it is consistent to have off-shell backgrounds on which one can have whatever theory describing the propagation and interactions of fluctuating modes. We only want these fluctuations to be small (kind of a probe theory) to not influence the background too much (backreaction is neglected). For example, we can study the quantum fluctuations around the flat background in a theory that incorporates the cosmological constant term, too. Indeed, we can always consider contributions to the propagator and 
vertices coming from the cosmological constant term even on a flat spacetime background.

In this paper we show that the analysis of perturbative linear stability (equivalent to the analysis of the spectrum of linear perturbations around a given background) gives the same results as in the flat spacetime case, and hence these gravitational vacuum configurations are perfectly stable. There is still a question, what is the vacuum here: gravitational vacuum or vacuum with a value of the cosmological constant, or no gravitational field at all (flat Minkowski spacetime)? Besides this we think that having a one vacuum (which may even be false) is fine for having a good candidate for quantum gravitational fundamental theory. Moreover, the quantization around MSS may have very important meaning in nonsupersymmetric theories (in unbroken supergravity the vacuum must be flat due to constraints coming from the supersymmetry algebra), and it must be considered seriously like the quantization around flat spacetime.

Last but not least, we must look for a theory consistent on a MSS spacetime background because the cosmological observations suggest that we are living in an exponentially expanding de-Sitter-like universe. Despite that for all Earthand solar system-based gravitational experiments we can safely neglect the effect of being in the dS phase, it is crucial that the theory, which has very good quantum properties around the Minkowski background, can also be formulated around any other MSS without major obstructions. Hereby, we show that such a theory exists and is well defined, and it has the same analogous good quantum and UV properties as the theory previously studied on the flat spacetime background. The theory around any MSS possesses the following virtues: is generally covariant, background-independent, perturbatively unitary [15-18], and in the quantum domain can easily be selected to be superrenormalizable or UV finite. It is easily seen that the presence of one constant parameter (namely the cosmological constant) in this fundamental theory does not destroy, but rather only generalizes, the amazing structure already known around the flat background. Indeed, on a MSS the theory is only slightly modified with respect to the theory on the flat background. The MSS backgrounds are very well behaved: they are, for example, constant with respect to covariant derivatives, and the commutators of derivatives can be traced back to some correction proportional to $\Lambda_{\mathrm{cc}}$. Moreover, the background curvature tensors can be completely written out using only the metric tensor and the parameter $\Lambda_{\mathrm{cc}}$. Therefore, we are going to include the cosmological constant in all the operators present in the action. In particular, the form factors could be selected to be functions of $\Lambda_{\mathrm{cc}}$ or the Ricci scalar. In the former case, as an additional advantage we can easily recover the flat spacetime results, by taking the limit $\Lambda_{\mathrm{cc}} \rightarrow 0$.

At the level of classical solutions the gravitational potential in the class of nonlocal theories is singularityfree and approaches a constant at $r=0$, regardless of the particular form factor appearing in the action [19-30]. This was found in the context of approximate solutions. On the other hand, regular bouncing solutions and Starobinsky's cosmological solution have been shown to solve exactly the equations of motion of the nonlocal theory [31]. However, Ricci-flat spacetimes and the Friedmann-Robertson-Walker (FRW) spacetime in the presence of radiation are still exact solutions of the weakly nonlocal theory [32]. This issue also has to do with the question of localization of nonlocal theories as addressed in [33]. Therefore, any form of nonlocality is not enough to smear out the singularities. However, at the present stage we cannot exclude that a special nonlocal theory could have only nonsingular solutions. Moreover, we have evidences that in this class of theories with infinitely many derivatives the black hole entanglement entropy is completely regularized and takes only finite values $[13,34]$.

In Sec. II we review the perturbative weakly nonlocal gravitational theory around the Minkowski space: the propagator, power counting, superrenormalizability, and finiteness at quantum level. In Sec. III we propose two classes of weakly nonlocal theories on (A)dS, and we explicitly prove that the action at the second order in the graviton fluctuations has the same structure of the Einstein-Hilbert one. In Sec. IV we show that the theory is finite at quantum level, while in Sec. V we prove that for one out of the two classes of theories a perturbative field redefinition allows one to map the nonlocal theory into the Einstein-Hilbert theory plus cosmological constant. In the last section we propose and study the most general weakly nonlocal field theory.

Most of the results obtained in this paper can easily be exported to Lee-Wick gravitational theories [35-40] just by replacing the nonlocal form factors with appropriate polynomials.

\section{NONLOCAL GRAVITATIONAL THEORIES ON MINKOWSKI VACUUM}

The most general $D$-dimensional theory weakly nonlocal (or quasilocal) and quadratic in curvature reads [1-11]

$$
\begin{aligned}
\mathcal{L}_{\mathrm{g}}= & -2 \kappa_{D}^{-2} \sqrt{|g|}\left[R+R \gamma_{0}(\square) R+\mathbf{R i c} \gamma_{2}(\square) \mathbf{R i c}\right. \\
& \left.+\mathbf{R i e m} \gamma_{4}(\square) \mathbf{R i e m}+\mathcal{V}\right] .
\end{aligned}
$$

The above Lagrangian density of the theory consists of a kinetic weakly nonlocal operator quadratic in curvature, three entire functions $\gamma_{0}(\square), \gamma_{2}(\square), \gamma_{4}(\square)$, and a set of local terms $\mathcal{V}$ cubic or higher in curvature. ${ }^{2}$ The latter consists of operators with a properly chosen number of derivatives to not spoil the good quantum properties of

\footnotetext{
${ }^{2}$ Definitions. - The metric tensor $g_{\mu \nu}$ has signature $(-+\ldots+)$ and the curvature tensors are defined as follows: $R^{\mu}{ }_{\nu \rho \sigma}=$ $-\partial_{\sigma} \Gamma_{\nu \rho}^{\mu}+\ldots, R_{\mu \nu}=R^{\rho}{ }_{\mu \rho \nu}, R=g^{\mu \nu} R_{\mu \nu}$. With symbol $\mathcal{R}$ we generally denote one of the above curvature tensors.
} 
the theory. Moreover, $\square=g^{\mu \nu} \nabla_{\mu} \nabla_{\nu}$ is the covariant d'Alembertian (or box) operator, while the entire functions $\gamma_{\ell}(\square)$ are defined in terms of exponentials of entire functions $H_{\ell}(z)(\ell=0,2)$, namely

$$
\gamma_{0}(\square)=-\frac{(D-2)\left(e^{H_{0}(\square)}-1\right)+D\left(e^{H_{2}(\square)}-1\right)}{4(D-1) \square}+\gamma_{4}(\square),
$$

$\gamma_{2}(\square)=\frac{e^{H_{2}(\square)}-1}{\square}-4 \gamma_{4}(\square)$,

while $\gamma_{4}(\square)$ stays arbitrary. It is only constrained by renormalizability to have the same asymptotic UV behavior as the other two form factors $\gamma_{\ell}(\square)(\ell=0,2)$. The minimal choice compatible with unitarity and superrenormalizability corresponds to retaining only two out of three form factors; i.e., we can choose $\gamma_{4}(\square)=0$.

Finally, the entire functions $V_{\ell}^{-1}(z) \equiv \exp \left(H_{\ell}(z)\right)$ ( $z \equiv-\square_{\Lambda} \equiv-\square / \Lambda^{2}$ ) (for $\ell=0,2$ ) introduced in (2) and (3) satisfy the following general conditions [3,41]:

(i) $V_{\ell}^{-1}(z)$ is real and positive on the real axis, and it has no zeros on the whole complex plane $|z|<+\infty$. This requirement implies that there are no gaugeinvariant poles other than the transverse massless physical graviton pole.

(ii) $\left|V_{\ell}^{-1}(z)\right|$ has the same asymptotic behavior along the real axis at $\pm \infty$.

(iii) There exist $\Theta>0, \Theta<\pi / 2$, and positive integer $\gamma$, such that asymptotically

$\left|V_{\ell}^{-1}(z)\right| \rightarrow|z|^{\gamma+\mathrm{N}+1}, \quad$ when $|z| \rightarrow+\infty$

with $\gamma \geq \frac{D_{\text {even }}}{2} \quad$ or $\quad \gamma>\frac{D_{\text {odd }}-1}{2}$,

for the complex values of $z$ in the conical regions $C$ defined by

$$
C=\{z \mid-\Theta<\arg z<+\Theta, \pi-\Theta<\arg z<\pi+\Theta\} .
$$

The last condition is necessary to achieve the maximum convergence of the theory in the UV regime. The necessary asymptotic behavior is imposed not only on the real axis but also on the conical regions that surround it. In an Euclidean spacetime, the condition (ii) is not strictly necessary if (iii) applies. In (4) the capital $\mathrm{N}$ is defined to be the following function of the spacetime dimension $D$ : $2 \mathrm{~N}+4=D_{\text {odd }}+1$ in odd dimensions and $2 \mathrm{~N}+4=$ $D_{\text {even }}$ in even dimensions. Moreover, by $\Lambda$ we denote the scale of nonlocality of the theory (not to be confused with the cosmological constant: $\Lambda_{\text {cc }}$ ).

One example of an entire function due to Tomboulis [3] is

$$
V^{-1}(z)=e^{\frac{1}{2}\left[\Gamma\left(0, p(z)^{2}\right)+\gamma_{E}+\log \left(p(z)^{2}\right)\right]},
$$

where $\Gamma(0, x)$ is the incomplete Gamma function with its first argument put to zero, $p(z)$ is a polynomial of degree $\gamma+\mathrm{N}+1$ and $\gamma_{E}$ is the Euler-Mascheroni mathematical constant. To achieve (super-)renormalizability the degrees of the polynomials appearing in the definitions of $V_{0}^{-1}(z)$ and $V_{2}^{-1}(z)$ must be equal. In the rest of the paper we will denote the common degree by $\gamma+\mathrm{N}+1(N=0$ in $D=4)$.

A few comments are in order here:

(i) First, it is obvious that the Minkowski spacetime is indeed a solution of the background equations of motion (EOM) corresponding to the above action (1). Other terms than the Einstein-Hilbert one in the original Lagrangian are at least quadratic in curvature and as such vanish when evaluated for the Minkowski metric. Even though the EOM are not used in our present analysis, the reader can find them in $[32,42,43]$. A cosmological constant term cannot be introduced here as it would lead to a constant nontrivial curvature at least.

(ii) Second, the action (1) is written exactly in the form as it is above because below we want to highlight the structure of the gravity propagator. Since the propagator can be read from a quadratic variation of the background action, we worry about terms at most quadratic in curvatures. Higher curvature corrections vanish upon the second variation as long as the background curvature itself is zero (as it is the case in the Minkowski flat background).

(iii) The requirement that the form factors $\gamma_{\ell}$ are entire functions deserves a little bit more explanation. The objects of our consideration are weakly nonlocal theories. This means that we have an analytic function in the whole complex plane with, in particular, a smooth limit when momenta tend to zero. The way to think about this is to introduce a scale of gravity modification $\Lambda$ with the dimension of mass and carefully write everywhere $\square / \Lambda^{2}$. As such the low energy limit is when the nonlocality scale goes to infinity. From here we find out that the form factors must be at least analytic in the origin. One may wonder why we need them to be entire functions, i.e., analytic everywhere. It can be shown that the propagators of canonical variables in Arnowitt-DeserMisner (ADM) formalism (which are observable quantities during inflation, for instance) feature a propagator with the form factor $\gamma_{0}$ in the denominator. As such, if the function $\gamma_{0}$ has some pole, it will become a new pole for the canonical variable and the quantum properties of the theory would be spoiled with respect to our expectations for the measurements. The mathematical details of these arguments can be found in a parallel study [44]. However, we make a statement that indeed the functions $\gamma_{\ell}$ must be entire.

(iv) The advertised above form of the form factors $\gamma_{\ell}$ and the comment that only two out of three of these 
functions are essential is a consequence of the structure of the propagator, and this is the matter of the succeeding analysis. It is, however, worth mentioning that the formulas (2) and (3) do not guarantee that $\gamma_{\ell}$ are entire functions even though the functions $H_{0,2}$ are. This should be checked independently.

We additionally remark here the reason to call the term $\mathcal{V}$ appearing in the action (1) "curvature potential." First of all, we argue that for any gauge theory as well as for gravity the strict distinction between the kinetic term and the potential of interaction does not exist. This is due to gauge invariance that connects interactions also with standard terms responsible for the propagator. The nomenclature we have adopted here is that by kinetic terms we mean terms that do contribute to the propagator around flat spacetime. Around the flat spacetime typically the kinetic terms are operators up to quadratic in curvature, while in the "curvature potential" we put all the terms cubic and higher in the curvature. The counting above is insensitive to the number of covariant derivatives appearing in the term under consideration. This is the only meaningful difference between the two parts of the action. On MSS operators cubic and higher in the curvature can contribute to the propagator. However, we can suitably modify the potential to make it compatible with the above definition around the Minkowski flat spacetime. Moreover, the locality of $\mathcal{V}$ is not a must, while the weak nonlocality is not required by the unitarity.

Finally, since in the gravitational case the notion of local energy density of the gravitational field is not well defined (strictly this is not a gauge-invariant observable with respect to the diffeomorphism group), we cannot sensibly speak about the potential energy for the gravitational Lagrangian case. We want to emphasize that even in the case of finite QED (studied in [45]) the role of the potential $\mathcal{V}$ is different from the standard role ascribed to it in classical mechanics or in other field theory models, so it is a little inappropriate to call it that.

\section{A. Propagator and unitarity around the Minkowski spacetime}

Splitting the spacetime metric into the flat Minkowski background and the dimensionful fluctuation $h_{\mu \nu}$ defined by $g_{\mu \nu}=\eta_{\mu \nu}+\kappa_{D} h_{\mu \nu}$ (here and above $\kappa_{D}$ is proportional to the square root of the gravitational Newton constant), we can expand the action (1) to the second order in $h_{\mu \nu}$. The result of this expansion together with the usual harmonic gauge-fixing term reads [46]

$$
\mathcal{L}_{\text {quad }}+\mathcal{L}_{\mathrm{GF}}=\frac{1}{2} h^{\mu \nu} \mathcal{O}_{\mu \nu, \rho \sigma} h^{\rho \sigma},
$$

where the operator $\mathcal{O}$ is made out of two terms, one coming from the quadratization of (1) and the other from the following gauge-fixing term, $\mathcal{L}_{\mathrm{GF}}=$ $\xi^{-1} \partial^{\nu} h_{\mu \nu} \omega\left(-\square_{\Lambda}\right) \partial_{\rho} h^{\rho \mu}$, where $\omega\left(-\square_{\Lambda}\right)$ is a weight functional $[47,48]$. The d'Alembertian operator in $\mathcal{L}_{\text {quad }}$ and the gauge-fixing term must be conceived on the flat spacetime. Inverting the operator $\mathcal{O}$ [46] and making use of the form factors (2) and (3), we find the two-point function in the harmonic gauge $\left(\partial^{\mu} h_{\mu \nu}=0\right)$,

$\mathcal{O}^{-1}=\frac{\xi\left(2 P^{(1)}+\bar{P}^{(0)}\right)}{2 k^{2} \omega\left(k^{2} / \Lambda^{2}\right)}+\frac{P^{(2)}}{k^{2} e^{H_{2}\left(k^{2} / \Lambda^{2}\right)}}-\frac{P^{(0)}}{(D-2) k^{2} e^{H_{0}\left(k^{2} / \Lambda^{2}\right)}}$.

We omitted the tensorial indices for the propagator $\mathcal{O}^{-1}$ and the projectors $\left\{P^{(0)}, P^{(2)}, P^{(1)}, \bar{P}^{(0)}\right\}$ defined in $[46,49]{ }^{3}$

The propagator (7) is the most general one compatible with unitarity. It propagates no other degree of freedom (d.o.f.) besides the standard massless transverse spin-2 graviton. This follows from the fact that exponents of entire functions are special entire functions with no zeros. So we technically avoid new poles, which means we avoid new physical d.o.f. Returning to the comment in the previous subsection we see that the structure of the propagator advocates the form of form factors $\gamma_{\ell}$ as the absence of new d.o.f. was exactly the requirement behind formulas (2) and (3). We also note that in order to have a well behaved propagator we need to get a correct form of only two factors corresponding to spin-0 and spin-2 parts. This explains why one function out of three $\gamma_{\ell}$ can be put to zero from the point of view of unitarity. Further, the unitarity is manifest, because the optical theorem at tree level is trivially satisfied, namely

$$
\begin{aligned}
& 2 \operatorname{Im}\left\{T(k)^{\mu \nu} \mathcal{O}_{\mu \nu, \rho \sigma}^{-1} T(k)^{\rho \sigma}\right\} \\
& \quad=\left.2 \pi \operatorname{Res}\left\{T(k)^{\mu \nu} \mathcal{O}_{\mu \nu, \rho \sigma}^{-1} T(k)^{\rho \sigma}\right\}\right|_{k^{2}=0}>0,
\end{aligned}
$$

where $T^{\mu \nu}(k)$ is the Fourier transform of the conserved energy tensor of a matter source.

So far we have proved that the theory is unitary at the perturbative level around the Minkowski spacetime. However, we will probably be able to prove the nonperturbative unitarity of the theory around the Minkowski

${ }^{3}$ The standard projectors are defined by

$$
\begin{aligned}
P_{\mu \nu, \rho \sigma}^{(2)}(k) & =\frac{1}{2}\left(\theta_{\mu \rho} \theta_{\nu \sigma}+\theta_{\mu \sigma} \theta_{\nu \rho}\right)-\frac{1}{D-1} \theta_{\mu \nu} \theta_{\rho \sigma}, \\
P_{\mu \nu, \rho \sigma}^{(1)}(k) & =\frac{1}{2}\left(\theta_{\mu \rho} \omega_{\nu \sigma}+\theta_{\mu \sigma} \omega_{\nu \rho}+\theta_{\nu \rho} \omega_{\mu \sigma}+\theta_{\nu \sigma} \omega_{\mu \rho}\right), \\
P_{\mu \nu, \rho \sigma}^{(0)}(k) & =\frac{1}{D-1} \theta_{\mu \nu} \theta_{\rho \sigma}, \quad \bar{P}_{\mu \nu, \rho \sigma}^{(0)}(k)=\omega_{\mu \nu} \omega_{\rho \sigma}, \\
\theta_{\mu \nu} & =\eta_{\mu \nu}-\frac{k_{\mu} k_{\nu}}{k^{2}}, \quad \omega_{\mu \nu}=\frac{k_{\mu} k_{\nu}}{k^{2}} .
\end{aligned}
$$

We have also replaced $-\square$ by $k^{2}$ in the quadratized action. 
spacetime in the near future. On the other hand, unitarity around general backgrounds is a very difficult task. Indeed, unitarity is only well defined in Minkowski spacetime mainly because it is not clear how to define the concept of a particle in curved spacetime and even how unambiguously to define the asymptotic states for the scattering $S$ matrix. We remind the reader that we can sensibly speak about the unitarity of the $S$ matrix only in theories where we have a well defined $S$ matrix. Regarding issues related to unitarity, at most what can be proved is the absence of ghosts on any background for some special theories (for example, Einstein gravity is ghost-free around any background). Nevertheless, in a recent paper we proved that we can have up to 8 d.o.f. in nonlocal gravity on a general background, and we do not know if some of them are ghostlike. In this paper we are going to prove that there are no ghosts in AdS and dS spacetimes (Sec. V), while in two other papers [50,51] we have proved the absence of ghosts (actually linear stability) around Ricci-flat spacetimes. However, the linear stability of a general background is really a difficult task and beyond the scope of this paper.

\section{B. Power counting in a nutshell}

We now review $[1,3,5,6,52,53]$ the power counting analysis of the quantum divergences. We remark that the divergences do not depend on the choice of the background spacetime metric; therefore, the results in this subsection apply equally well to the case of theories studied around general MSS backgrounds. In the high energy regime, the above propagator (7) in momentum space schematically scales as

$$
\mathcal{O}^{-1}(k) \sim \frac{1}{k^{2 \gamma+D}} \quad \text { in the UV. }
$$

The vertices can be collected in different sets that may or may not involve the entire functions $\exp H_{\ell}(z)$. However, to find a bound on the quantum divergences it is sufficient to concentrate on the leading operators in the UV regime. These operators scale as the inverse of the propagator giving the following upper bounds on the superficial degree of divergence of any graph $G[1,3,5,6]$,

$$
\omega(G)=D L+(V-I)(2 \gamma+D),
$$

in a spacetime of even or odd dimension, respectively. We simplify the above relation further to

$$
\omega(G)=D-2 \gamma(L-1) .
$$

In (12), we used the topological relation between the number of vertices $V$, the number of internal lines $I$, and the number of loops $L: I=V+L-1$. Thus, if $\gamma>D / 2$, in the theory only one-loop divergences survive. Therefore, the theory is superrenormalizable $[1,3,5,6,53]$ and only a finite number of operators of mass dimension up to $M^{D}$ has to be included in the action in the even dimension for the purpose of renormalization.

Notice that the power counting analysis can be done in Minkowski spacetime because any smooth spacetime is locally flat and the divergences are related to the UV coincidence limit in the correlation functions. Therefore, we can expand around whatever background, and we will always end up with the same divergent contributions to the quantum effective action; namely we will always get the same beta functions.

\section{The theory in Weyl basis}

We can equally consider a different action, which will be written by reshuffling quadratic in curvature terms in (1). The following action is equivalent to (1) for everything about unitarity [the propagator is given again by (7)] and superrenormalizability or UV finiteness and its Lagrangian density reads

$$
\begin{aligned}
\mathcal{L}_{\mathrm{C}}= & -2 \kappa_{D}^{-2} \sqrt{|g|}\left[R+\mathbf{C} \gamma_{\mathrm{C}}(\square) \mathbf{C}+R \gamma_{\mathrm{S}}(\square) R\right. \\
& \left.+\mathbf{R i e m} \gamma_{\mathrm{R}}(\square) \mathbf{R i e m}+\mathcal{V}\right], \\
\gamma_{\mathrm{C}}= & -\frac{D-2}{4} \gamma_{2}, \quad \gamma_{\mathrm{S}}=\gamma_{0}+\frac{1}{2(D-1)} \gamma_{2}, \\
\gamma_{\mathrm{R}}= & \gamma_{4}+\frac{D-2}{4} \gamma_{2},
\end{aligned}
$$

where $\mathbf{C}$ is the Weyl tensor and all the form factors $\gamma_{\ell}$ are defined in (2) and (3).

To start with, we recall that only two form factors are needed to have an appropriate propagator. We thus put $\gamma_{\mathrm{R}}=0$ and the theory (13) reduces to

$\mathcal{L}_{\mathrm{C}}=-2 \kappa_{D}^{-2} \sqrt{|g|}\left[R+\mathbf{C} \gamma_{\mathrm{C}}(\square) \mathbf{C}+R \gamma_{\mathrm{S}}(\square) R+\mathcal{V}(\mathbf{C})\right]$,

$\gamma_{\mathrm{C}}=\frac{D-2}{4(D-3)} \frac{e^{H_{2}}-1}{\square}, \quad \gamma_{\mathrm{S}}=-\frac{D-2}{4(D-1)} \frac{e^{H_{0}}-1}{\square}$.

In $D=4$ it is enough to include $\mathcal{V}$ made out of two Weyl killers to end up with a completely finite quantum gravitational theory at any perturbative order in the loop expansion. For example, we can choose the following two operators:

$$
\begin{aligned}
\mathcal{V}(\mathbf{C})= & s_{w}^{(1)} C_{\mu \nu \rho \sigma} C^{\mu \nu \rho \sigma} \square^{\gamma-2} C_{\alpha \beta \gamma \delta} C^{\alpha \beta \gamma \delta} \\
& +s_{w}^{(2)} C_{\mu \nu \rho \sigma} C^{\alpha \beta \gamma \delta} \square^{\gamma-2} C_{\alpha \beta \gamma \delta} C^{\mu \nu \rho \sigma} .
\end{aligned}
$$

The Gauss-Bonnet (GB) operator does not contribute to the divergent part of the quantum effective action in $D=4$ when the manifold has a trivial topology; namely the spacetime is topologically equivalent to the Minkowski 
one or the Euclidean space (see, for example, [1]). However, in the rest of the paper we will deal with the (A)dS space, and we will have to take care of the divergence proportional to the GB, too.

The beta functions for the two couplings in front of terms quadratic in curvature can be only linear in the front coefficients $s_{w}^{(1)}$ and $s_{w}^{(2)}[6]$, and then we can always find a solution to the equations $\beta_{R^{2}}=0$ and $\beta_{\mathbf{R i c}^{2}}=0$ regardless of the energy scale and the loop order. The integral of the Gauss-Bonet operator is in this section identically zero because we assume the space to be topologically equivalent to the Minkowski spacetime. Later we will be forced to give up this hypothesis in (A)dS.

As pointed out in the Introduction the weak nonlocality is not sufficient to solve the singularity issue that plagues the Einstein-Hilbert gravitational theory. In particular, for the theory in the Weyl basis presented in this section the FRW metrics for conformal matter $\left(T_{\text {matter }} \equiv 0\right)$ solve exactly the nonlocal EOM [32]. This means that the bigbang singularity shows up in an exact solution of our nonlocal quantum gravity. However, if the gravitational sector also enjoys conformal invariance, then any FRW singular spacetime is conformally equivalent to the flat spacetime by a conformal rescaling and the singularity turns out to be unphysical [54]. Notice that the presence of singularities in particular nonlocal theories does not rule out that it may exist a nonlocal theory, which is singularityfree. However, the naive nonlocality by itself is not enough $[32,54]$. The crucial ingredient here is the conformal symmetry, which allows for rescalings such as those described above. Moreover, scale invariance helps with the singularity of geodesics $[54,55]$ and also with some issues of black hole physics [56,57].

\section{NONLOCAL GRAVITY IN (A)dS VACUUM}

A generalization to a constant curvature background is rather straightforward. We here provide the expansion of the action to the second order in the gravitational fluctuations around an (A)dS spacetime, and we will infer about the stability properties of the theory around any maximally symmetric vacuum. We retrace the path followed for the case of the Minkowski vacuum to mimic as much as possible the Einstein-Hilbert theory. Therefore, we will end up with a quadratic operator that reproduces the one from Einstein's gravity on the same background up to, at most, two multiplicative form factors that do not change the structure of the classical two-point function [58]. Technically, we will use the previous computations published in $[31,59,60]$ (see also Appendix). For definiteness we will first concentrate on the situation on on-shell MSS backgrounds. For them we can use that $a_{\Lambda}=\Lambda_{\mathrm{cc}}$.

For reasons that will be clear later in the paper, we here study two classes of theories that we identify as theories in the "Weyl basis" and theories in the "Ricci basis."

\section{A. A class of theories in the Weyl basis}

To see how things work we stick to $D=4$, make use of the Weyl basis, and consider the case $\gamma_{R}=0$,

$$
\begin{aligned}
& \mathcal{L}_{\mathrm{CR}}=-2 \kappa_{4}^{-2} \sqrt{|g|}\left[R-2 \Lambda_{\mathrm{cc}}+\mathbf{C} \gamma_{\mathrm{C}}(\square) \mathbf{C}+R \gamma_{\mathrm{S}}(\square) R+\mathcal{V}(\mathbf{C})\right] \text { with } \\
& \gamma_{\mathrm{C}}(\square)=\frac{1}{2}\left(e^{H_{2}\left(\square-\frac{2}{3} R\right)}-1\right) \frac{1}{\square-\frac{2}{3} R}, \quad \gamma_{\mathrm{S}}(\square)=-\frac{1}{6} \frac{1}{\square+\frac{R}{3}}\left(e^{H_{0}(\square, R)}-1\right),
\end{aligned}
$$

where the translations of the covariant box operators (by the amount proportional to $R$ ) in comparison to the form factors given in (16) will be clear shortly. Notice that the form factors (19) turn in (16) when the formal limit $R \rightarrow 0$ in (19) is taken. Moreover, the ordering of the operators could be relevant in (19) for some choices of the asymptotic polynomials. Indeed, the arguments of the entire functions $\mathrm{H}_{0}$ and/or $\mathrm{H}_{2}$ can in general differ from the denominators in (19) so that they do not commute due to the Ricci scalar curvatures appearing with different numerical coefficients. ${ }^{4}$

${ }^{4}$ Another slightly different choice of the form factors with respect to (19) can make irrelevant the ordering, namely

$$
\gamma_{\mathrm{C}}(\square)=\frac{1}{2} \frac{e^{H_{2}\left(\square-\frac{8}{3} \Lambda_{\mathrm{cc}}\right)}-1}{\square-\frac{8}{3} \Lambda_{\mathrm{cc}}}, \quad \gamma_{\mathrm{S}}(\square)=-\frac{1}{6} \frac{e^{H_{0}\left(\square+\frac{4}{3} \Lambda_{\mathrm{cc}}\right)}-1}{\square+\frac{4}{3} \Lambda_{\mathrm{cc}}},
$$

where we replaced $R$ with the cosmological constant that now appears not only in the local Einstein-Hilbert sector of the theory but also explicitly in the form factors. Notice that this is an off-shell replacement, and it is just a different definition of the theory. Here the amount of the shift in terms of the cosmological constant had been fixed in order to have stability around the (A)dS spacetime. Moreover, the form factors (20) can easily be expressed as $\sum_{r=0}^{\infty} a_{r} \square^{r}$ for a proper choice of the coefficients $a_{r}$ because $\Lambda_{\mathrm{cc}}$ is a constant and then the form factor is commutative contrary to the one in the main text, namely (19). Therefore, it is easy to implement the power counting analysis developed in $[1,3,52]$. The vertices for the theory with form factors $(20)$ will contain the incremental ratios defined in [52] for the same form factors (20) with $\square$ replaced with $\square_{M}$ (the box operator on Minkowski space) (see also the discussion in the last part of this subsection). 
In taking the quadratic part of the action (18) in the graviton fluctuation $h_{\mu \nu}$ we use the following decomposition of the graviton field:

$h_{\mu \nu}=h_{\mu \nu}^{\perp}+\nabla_{(\mu} A_{\nu)}^{\perp}+\left(\nabla_{\mu} \nabla_{\nu}-\frac{1}{4} g_{\mu \nu} \square\right) B+\frac{1}{4} g_{\mu \nu} h$,

where the spin-two fluctuation $h_{\mu \nu}^{\perp}$ contains 5 d.o.f. because it satisfies $\nabla^{\mu} h_{\mu \nu}^{\perp}=g^{\mu \nu} h_{\mu \nu}^{\perp}=0$. The transverse vector $A_{\nu}^{\perp}$, satisfying $\nabla^{\mu} A_{\mu}^{\perp}=0$, accounts for 3 d.o.f. Finally, $B$ and $h$ are two real scalars. However, $A_{\mu}^{\perp}$ automatically drops out of the second variation of the action, and out of the two scalars only the following combination $\phi=\square B-h$ appears there.

We end up with the following second order variation of the action $[31,59,60]$ :

$$
\begin{aligned}
S_{(\mathrm{A}) \mathrm{dS}}^{(2) \mathrm{CR}} & =\frac{1}{2} \int d^{4} x \sqrt{|\bar{g}|}\left\{\tilde{h}^{\perp \mu \nu}\left(\square-\frac{\bar{R}}{6}\right)\right. \\
& \times\left[1+2 \gamma_{\mathrm{S}}(0) \bar{R}+2\left(\square-\frac{\bar{R}}{3}\right) \gamma_{\mathrm{C}}\left(\square+\frac{\bar{R}}{3}\right)\right] \tilde{h}_{\mu \nu}^{\perp} \\
& \left.-\tilde{\phi}\left(\square+\frac{\bar{R}}{3}\right)\left[1+2 \gamma_{\mathrm{S}}(0) \bar{R}-6\left(\square+\frac{\bar{R}}{3}\right) \gamma_{\mathrm{S}}(\square)\right] \tilde{\phi}\right\},
\end{aligned}
$$

where we introduced the canonically normalized fields $\tilde{h}_{\mu \nu}^{\perp}=M_{\mathrm{P}} h_{\mu \nu}^{\perp} / 2, \tilde{\phi}=\sqrt{3 / 32} M_{\mathrm{P}} \phi$, and $M_{\mathrm{P}}^{2}=4 \kappa_{4}^{-2}$. In this part of the section the bar operators $\overline{\mathcal{O}}$ denote any background quantity. Moreover, $\gamma_{S}(0)$ can be read out of the following general expansion:

$\gamma_{\mathrm{S}}(\square)=\sum_{i=0}^{+\infty} c_{\mathrm{S}, i}\left[(\square+X)^{n_{2}}\right]^{i}\left(\square^{n_{1}}\right)^{i}, \quad n_{1}, n_{2} \in \mathbb{N}$,

where $X$ is an operator proportional to the background Ricci scalar $R$. The chosen order in (23) is consistent with the polynomial given below in (26). The detailed expressions for the most general second order variations of various actions on MSS are collected in Appendix. We can assume $\gamma_{\mathrm{S}}(0)=0$ [i.e., $c_{\mathrm{S}, 0}=0$ in (19), (23)] because this is consistent with the requirements for the special entire function $H(z)$ [3]. Therefore, replacing the form factors (19) in the variation (22) we end up with the following result:

$$
\begin{aligned}
S_{(\mathrm{A}) \mathrm{dS}}^{(2) \mathrm{CR}}= & \frac{1}{2} \int d^{4} x \sqrt{|\bar{g}|}\left\{\tilde{h}^{\perp \mu \nu}\left(\square-\frac{\bar{R}}{6}\right) e^{H_{2}\left(\square-\frac{\bar{R}}{3}\right)} \tilde{h}_{\mu \nu}^{\perp}\right. \\
& \left.-\tilde{\phi}\left(\square+\frac{\bar{R}}{3}\right) e^{H_{0}(\square, \bar{R})} \tilde{\phi}\right\} .
\end{aligned}
$$

The condition $\gamma_{\mathrm{S}}(0)=0$ and the locality of counterterms force us to select the following entire function (we here consider the $\gamma=3$ case):

$H_{0}(\square, R)=\frac{1}{2}\left\{\gamma_{E}+\Gamma\left(0,\left[p_{\mathrm{S}}(\square, R)\right]^{2}\right)+\log \left[p_{\mathrm{S}}(\square, R)\right]^{2}\right\}$,

$p_{\mathrm{S}}(\square, R)=\frac{1}{\Lambda^{8}}\left(\square+\frac{R}{3}\right)^{2} \square^{2}$.

For the form factor $\gamma_{C}$ we can take the following entire function $\mathrm{H}_{2}$ :

$$
\begin{aligned}
H_{2}\left(\square-\frac{2}{3} R\right)= & \frac{1}{2}\left\{\gamma_{E}+\Gamma\left(0,\left[p_{\mathrm{C}}\left(\square-\frac{2}{3} R\right)\right]^{2}\right)\right. \\
& \left.+\log \left[p_{\mathrm{C}}\left(\square-\frac{2}{3} R\right)\right]^{2}\right\}, \\
p_{\mathrm{C}}\left(\square-\frac{2}{3} R\right)= & \frac{1}{\Lambda^{8}}\left(\square-\frac{2}{3} R\right)^{4} .
\end{aligned}
$$

We notice here that the choice of polynomials is fixed only by the UV behavior of the propagator and as such we have a lot of freedom in choosing them as long as basic principles are obeyed.

For instance, we can have "commutative" form factors (20) such that

$$
\begin{aligned}
& p_{\mathrm{C}}\left(\square, \Lambda_{\mathrm{cc}}\right)=\frac{1}{\Lambda^{8}} \square^{2}\left(\square-\frac{8}{3} \Lambda_{\mathrm{cc}}\right)^{2}, \\
& p_{\mathrm{S}}\left(\square, \Lambda_{\mathrm{cc}}\right)=\frac{1}{\Lambda^{8}} \square^{2}\left(\square+\frac{4}{3} \Lambda_{\mathrm{cc}}\right)^{2} .
\end{aligned}
$$

Notice that the above polynomials are zero for $\square=0$, which is crucial to secure $\gamma_{S}(0)=0$. Indeed, (29) is a polynomial in $\square$, and if we do not multiply by $\square^{2}$, we get a constant dimensionless contribution proportional to $\left(\Lambda_{\mathrm{cc}} / \Lambda^{2}\right)^{2} \propto c_{\mathrm{S}, 0} \neq 0$.

The following special choice of the polynomial $p_{\gamma+1}$ $(\gamma+1=8)$ makes also consistent the identification of $\mathrm{H}_{2}$ with $H_{0}$ :

$$
p_{8}(\square, R)=\frac{1}{\Lambda^{16}}\left(\square+\frac{R}{3}\right)^{2} \square^{2}\left(\square-\frac{2}{3} R\right)^{4} .
$$

It is now clear why the fraction $1 /\left(\square-\frac{2}{3} R\right)$ is located on the right in the definition of $\gamma_{C}(\square)$ in (19). This in 
turn results in that the second variation of the action simplifies to

$$
\begin{aligned}
S_{(\mathrm{A}) \mathrm{dS}}^{(2) \mathrm{CR}}= & \frac{1}{2} \int d^{4} x \sqrt{|\bar{g}|}\left\{\tilde{h}^{\perp \mu \nu}\left(\square-\frac{\bar{R}}{6}\right) e^{H_{8}\left(\square+\frac{\bar{R}}{3}\right)} \tilde{h}_{\mu \nu}^{\perp}\right. \\
& \left.-\tilde{\phi}\left(\square+\frac{\bar{R}}{3}\right) e^{H_{8}\left(\square+\frac{\bar{R}}{3}\right)} \tilde{\phi}\right\},
\end{aligned}
$$

where $H_{8}$ is in the class of entire functions (5) with the polynomial $p(z)$ substituted by the above definition of the polynomial $p_{8}(\square+\bar{R} / 3)$ evaluated on the (A)dS background of curvature $\bar{R}$.

With a globally well defined field redefinition we can now completely remove the form factor, and the kinetic operator turns into the one of Einstein-Hilbert theory with cosmological constant. The interactions will get modified by the field redefinition as well, but the Feynman diagrams will stay the same. However, in doing so we do not really need to equate the form factors in front of different spin modes. Therefore, for the moment the choice (30) is just to make the second order variation of the nonlocal theory as similar as we can to the Einstein-Hilbert one. Details about such a field redefinition are explained in Sec. V.

\section{Analysis of the "noncommutative" form factors}

In this quite technical subsection we study some properties of the form factors that will turn out to be crucial in Sec. IV about quantum finiteness. Let us remind the reader that the exponentials of one, two, or multiple matrices are defined by means of power series, namely

$$
\begin{aligned}
e^{X} & =\sum_{k=0}^{\infty} \frac{1}{k !} X^{k}, \\
e^{X+Y} & =\sum_{k=0}^{\infty} \frac{1}{k !}(X+Y)^{k}, \\
e^{X_{1}+X_{2}+X_{3}+\ldots+X_{N}} & =\sum_{k=0}^{\infty} \frac{1}{k !}\left(X_{1}+X_{2}+X_{3}+\ldots+X_{N}\right)^{k} .
\end{aligned}
$$

For the form factors defined in (19) with polynomials (26) and (27) we can make explicit the above formula (32) as follows:

$$
\begin{aligned}
e^{H_{0,2}(\square, R)}= & \sum_{n=0}^{\infty} \frac{1}{n !} H(\square, R)^{n} \\
= & \sum_{n=0}^{\infty} \frac{1}{n !}\left\{\frac { 1 } { 2 } \left[\Gamma\left(0, p_{\mathrm{S}, \mathrm{C}}(\square, R)^{2}\right)+\gamma_{E}\right.\right. \\
& \left.\left.+\log \left(p_{\mathrm{S}, \mathrm{C}}(\square, R)^{2}\right)\right]\right\}^{n} \\
= & \sum_{n=0}^{\infty} \frac{1}{n !}\left\{\sum_{m=1}^{\infty}(-1)^{m-1} \frac{1}{m !} \frac{p_{\mathrm{S}, \mathrm{C}}(\square, R)^{2 m}}{2 m}\right\}^{n} \\
= & \sum_{s=0}^{\infty} c_{s} p_{\mathrm{S}, \mathrm{C}}(\square, R)^{2 s},
\end{aligned}
$$

where the coefficients $c_{s}$ are obtained by comparing the last two sums above. Notice that $p(\square, R)$ certainly commutes with itself, but its arguments, namely $\square$ and $R$, do not commute. Therefore, it is still easily possible to apply the structure of the vertex functions found in [3] to the case of a binomial like (27) because the form factor $\gamma_{C}$ is a function of only one polynomial, namely

$$
\begin{aligned}
\gamma_{\mathrm{C}} & =\sum_{s=0}^{\infty} \tilde{c}_{s}\left(\square-\frac{2}{3} R\right)^{8 s-1} \\
& =\tilde{c}_{1}\left(\square-\frac{2}{3} R\right)^{7}+\tilde{c}_{3}\left(\square-\frac{2}{3} R\right)^{23}+\cdots \\
\text { or } \gamma_{\mathrm{C}} & =\sum_{r=0}^{\infty} a_{r}\left(\square-\frac{2}{3} R\right)^{r},
\end{aligned}
$$

for a proper and fixed choice of the coefficients $a_{r}$ given the coefficients $\tilde{c}_{s}$. Now we can apply the formula presented in [1] and rigorously proved in $[3,52]$ to the operator $\tilde{\square}=\square-2 R / 3$. In particular, we can introduce the following notation:

$$
\begin{aligned}
\gamma_{C} & =\sum_{r}^{\infty} a_{r}\left(\square_{M}+\mathcal{I}\right)^{r}, \\
\mathcal{I} & =\tilde{\square}-\square_{M}, \quad \square_{M}=\eta^{\mu \nu} \partial_{\mu} \partial_{\nu} .
\end{aligned}
$$

We also remind the reader that around fixed Minkowski background the form factor in momentum space is the Fourier transform of

$$
\gamma_{\mathrm{C}}=\sum_{r=0}^{\infty} a_{r}\left(\square_{M}\right)^{r}=\frac{1}{2} \frac{1}{\square_{M}}\left(e^{H_{0}\left(\square_{M}\right)}-1\right),
$$

while the gravitons' vertices come only from the perturbative expansion of $\mathcal{I}$. 
Less trivial is to apply the formula in [52] to $\gamma_{S}$ that we can express as follows:

$$
\begin{aligned}
\gamma_{\mathrm{S}} & =-\frac{1}{6} \frac{1}{\square+\frac{R}{3}} \sum_{s=1}^{\infty} c_{s} p_{\mathrm{S}}^{2 s}(\square, R)=-\frac{1}{6} \frac{1}{\square+\frac{R}{3}} \sum_{s=1}^{\infty} c_{s}\left(\frac{1}{\Lambda^{8}}\left(\square+\frac{R}{3}\right)^{2} \square^{2}\right)^{2 s} \\
& =-\frac{1}{6} \frac{1}{\square+\frac{R}{3}} \sum_{s=1}^{\infty} c_{s}\left(\frac{1}{\Lambda^{8}}\left(\square+\frac{R}{3}\right)^{2} \square^{2}\right)\left(\frac{1}{\Lambda^{8}}\left(\square+\frac{R}{3}\right)^{2} \square^{2}\right)^{2 s-1} \\
& =-\frac{1}{6}\left(\square^{3}+\frac{R}{3} \square^{2}\right) \sum_{s=1}^{\infty} \frac{c_{s}}{\Lambda^{16 s}}\left(\left(\square+\frac{R}{3}\right)^{2} \square^{2}\right)^{2 s-1}=-\frac{1}{6}\left(\square^{3}+\frac{R}{3} \square^{2}\right) \sum_{r=0}^{\infty} a_{r}\left(\left(\square+\frac{R}{3}\right)^{2} \square^{2}\right)^{r} .
\end{aligned}
$$

The coefficients $c_{s}$ are fixed using the definition (34), while the coefficients $a_{r}$ can be derived comparing the last two expressions in (38). Now we can apply the derivation in $[52]$ to

$$
\sum_{r=0}^{\infty} a_{r}\left(\square_{M}^{4}+\mathcal{I}\right)^{r}, \quad \mathcal{I}=\square^{4}-\square_{M}^{4}+O(R)
$$

When we expand in the graviton field we get interaction vertices coming from the variation of the binomial on the left of the sum in (38) and other vertices come from the variation of the sum. However, the full nonlocal contribution resulting from the variation of (38) will reconstruct the same incremental ratios as defined in [52], but for the form factor in Minkowski space rescaled by $1 / \square^{4}$, namely

$$
\frac{\gamma_{\mathrm{S}}\left(\square_{M}\right)}{\square_{M}^{4}}=-\frac{1}{6} \frac{e^{H_{0}\left(\square_{M}\right)}-1}{\square_{M}^{5}} \Lambda^{8} .
$$

We can forget the nonlocality to evaluate the divergent contributions to the quantum effective action.

\section{B. A class of theories in the Ricci basis}

As a second example we consider the following action involving the Ricci tensor, but not the Weyl tensor in the quadratic in curvature part of the action, namely

$$
\mathcal{L}_{\mathrm{SR}}=-2 \kappa_{4}^{-2} \sqrt{|g|}\left[R-2 \Lambda_{\mathrm{cc}}+\mathbf{S} \gamma_{\mathrm{S} 2}(\square) \mathbf{S}+R \gamma_{\mathrm{S}}(\square) R+\mathcal{V}(\mathbf{C})\right]
$$

where the rank-two tensor $\mathbf{S}$ is defined by

$$
S_{\mu \nu}=R_{\mu \nu}-\frac{1}{4} R g_{\mu \nu}
$$

In $D=4$ it is identically zero when evaluated on an (A)dS background, and, moreover, it is completely trace-free. The form factors in the action (41) are defined by

$$
\begin{aligned}
& \gamma_{\mathrm{S} 2}(\square)=\frac{1}{\square-\frac{R}{6}}\left(e^{H_{\mathrm{S} 2}\left(\left(\square-\frac{R}{6}\right)\left(\square-\frac{R}{3}\right)\right)}-1\right), \\
& \gamma_{\mathrm{S}}(\square)=-\frac{1}{6} \frac{1}{\square+\frac{R}{3}}\left(e^{H_{0}(\square, R)}-1\right)-\square \frac{1}{12\left(\square+\frac{R}{2}\right)}\left(e^{H_{\mathrm{S} 2}\left(\left(\square+\frac{R}{2}\right)\left(\square+\frac{R}{3}\right)\right)}-1\right) \frac{1}{\left(\square+\frac{R}{3}\right)} .
\end{aligned}
$$

We should here clarify how the entire functions defined above depend on their arguments. Let us start with $H_{S 2}$ in $\gamma_{\mathrm{S} 2}(\square)$, which is defined to be the following entire function of the polynomial $p_{\mathrm{S} 2}$ of a fourth degree in $\square$ :

$$
\begin{aligned}
H_{\mathrm{S} 2}\left(\left(\square-\frac{R}{6}\right)\left(\square-\frac{R}{3}\right)\right) & =\frac{1}{2}\left\{\gamma_{E}+\Gamma\left(0, p_{\mathrm{S} 2}^{2}(\square, R)\right)+\log \left(p_{\mathrm{S} 2}^{2}(\square, R)\right)\right\}, \\
p_{\mathrm{S} 2}(\square, R) & =\left(\square-\frac{R}{6}\right)^{2}\left(\square-\frac{R}{3}\right)^{2} .
\end{aligned}
$$


Therefore, the exponentiated entire function $H_{\mathrm{S} 2}\left(\left(\square+\frac{R}{3}\right)\left(\square+\frac{R}{2}\right)\right)$ in the second analytic operator in (44) is obtained translating the operator $\square$ by the amount $\frac{2}{3} R$, namely

$$
\begin{aligned}
& H_{\mathrm{S} 2}\left(\left(\square+\frac{R}{2}\right)\left(\square+\frac{R}{3}\right)\right) \\
& \quad:=\left.H_{\mathrm{S} 2}\left(\left(\square-\frac{R}{6}\right)\left(\square-\frac{R}{3}\right)\right)\right|_{\square \rightarrow \square+\frac{2}{3} R} .
\end{aligned}
$$

Notice how the translated $\square$ operators at the denominator in (44) have been placed in order to avoid ordering issues. ${ }^{5}$ Moreover, taking the "formal" limit $R \rightarrow 0$ in the form factors (44) and assuming $H_{\mathrm{S} 2}=H_{0}$ the above Lagrangian (41) turns into

$$
\begin{aligned}
& \mathcal{L}_{\mathrm{E}}=-2 \kappa_{D}^{-2} \sqrt{|g|}\left[R+G_{\mu \nu} \gamma_{\mathrm{G}}(\square) R^{\mu \nu}+\mathcal{V}\right], \\
& \gamma_{\mathrm{G}}=\frac{e^{H_{2}}-1}{\square}
\end{aligned}
$$

Finally, the second order variation of the action for the Lagrangian (41) reads

$$
\begin{aligned}
S_{(\mathrm{A}) \mathrm{dS}}^{(2) \mathrm{CSR}}= & \frac{1}{2} \int d^{4} x \sqrt{|\bar{g}|}\left\{\tilde{h}^{\perp \mu \nu}\left(\square-\frac{\bar{R}}{6}\right)\right. \\
& \times\left[1+2 \gamma_{\mathrm{S}}(0) \bar{R}+\left(\square-\frac{\bar{R}}{6}\right) \gamma_{\mathrm{S} 2}(\square)\right] \tilde{h}_{\mu \nu}^{\perp} \\
& -\tilde{\phi}\left(\square+\frac{\bar{R}}{3}\right)\left[1+2 \gamma_{\mathrm{S}}(0) \bar{R}-6\left(\square+\frac{\bar{R}}{3}\right) \gamma_{\mathrm{S}}(\square)\right. \\
& \left.\left.-\frac{1}{2} \square \gamma_{\mathrm{S} 2}\left(\square+\frac{2}{3} \bar{R}\right)\right] \tilde{\phi}\right\} .
\end{aligned}
$$

We also selected out a form factor such that $\gamma_{S}(0)=0$. For this purpose, after looking at the formula (44), it is sufficient to take the following asymptotic polynomial $p_{\gamma+1}$ (for $\left.\gamma+1=3+1\right)$ as an argument of $H_{0}$ :

$$
p_{\mathrm{S}}(\square, R)=\left(\square+\frac{R}{3}\right)^{2} \square^{2}
$$

\footnotetext{
${ }^{5} \mathrm{We}$ can use here different definitions of the form factors to avoid the ordering problems of the denominators versus the exponential form factors, namely

$$
\begin{aligned}
\gamma_{\mathrm{S} 2}(\square) & =\frac{e^{H_{\mathrm{S} 2}\left(\left(\square-\frac{4 \Lambda_{\mathrm{cc}}}{6}\right)\left(\square-\frac{4 \Lambda_{\mathrm{cc}}}{3}\right)\right.}-1}{\square-\frac{4 \Lambda_{\mathrm{cc}}}{6}}, \\
\gamma_{\mathrm{S}}(\square) & =-\frac{1}{6} \frac{e^{H_{0}\left(\square+\frac{4 \Lambda_{\mathrm{cc}}}{3}\right)}-1}{\square+\frac{4 \Lambda_{\mathrm{cc}}}{3}}-\square \frac{e^{H_{\mathrm{S} 2}\left(\left(\square+\frac{4 \Lambda_{\mathrm{cc}}}{3}\right)\left(\square+\frac{4 \Lambda_{\mathrm{cc}}}{2}\right)\right)}-1}{12\left(\square+\frac{4 \Lambda_{\mathrm{cc}}}{3}\right)\left(\square+\frac{4 \Lambda_{\mathrm{cc}}}{2}\right)} .
\end{aligned}
$$

The ordering is now irrelevant, because $\Lambda_{\mathrm{cc}}$ is a numerical constant.
}

Therefore, after plugging the form factors (44) in the second order variation (50) we end up again with (24), but with $H_{2}$ replaced by $H_{\mathrm{S} 2}$, namely

$$
\begin{aligned}
S_{(\mathrm{A}) \mathrm{dS}}^{(2) \mathrm{CR}}= & \frac{1}{2} \int d^{4} x \sqrt{|\bar{g}|}\left\{\tilde{h}^{\perp \mu \nu}\left(\square-\frac{\bar{R}}{6}\right) e^{H_{\mathrm{S} 2}\left(\left(\square-\frac{\bar{R}}{6}\right)\left(\square-\frac{\bar{R}}{3}\right)\right.} \tilde{h}_{\mu \nu}^{\perp}\right. \\
& \left.-\tilde{\phi}\left(\square+\frac{\bar{R}}{3}\right) e^{H_{0}(\square, \bar{R})} \tilde{\phi}\right\} .
\end{aligned}
$$

In order to end up with the same form factor in the spin-two as well as in the spin-zero graviton sectors we slightly modify the polynomial in (45) and we replace the curvature $R$ with the cosmological constant $\Lambda_{\text {cc }}$, namely

$$
\begin{aligned}
\tilde{p}\left(\square ; \Lambda_{\mathrm{cc}}\right) & =\left.\left(\square-\frac{R}{6}\right)^{2}\left(\square+\frac{R}{3}\right)^{2}\left(\square-\frac{R}{3}\right)^{2}\right|_{R \rightarrow 4 \Lambda_{\mathrm{cc}}} \times \square^{2} \\
& =\left(\square-\frac{2}{3} \Lambda_{\mathrm{cc}}\right)^{2}\left(\square+\frac{4}{3} \Lambda_{\mathrm{cc}}\right)^{2}\left(\square-\frac{4}{3} \Lambda_{\mathrm{cc}}\right)^{2} \square^{2},
\end{aligned}
$$

where we technically replaced the Ricci scalar $R$ with $4 \Lambda_{\mathrm{cc}}$ to end up with a form factor without ordering issues. This replacement does not mean that we evaluate the form factor on the background, but just that the form factor has a particular (a posteriori) dependence on the constant $\Lambda_{\mathrm{cc}}$. In (53) the untranslated $\square$ on the right secures that $\gamma_{S}(0)=0$. The form factors now read

$$
\begin{aligned}
\gamma_{\mathrm{S} 2}(\square)= & \frac{e^{H_{\mathrm{S} 2}\left(\tilde{p}\left(\left(\square-\frac{2}{3} \Lambda_{\mathrm{cc}}\right)\left(\square+\frac{4}{3} \Lambda_{\mathrm{cc}}\right)\left(\square-\frac{4}{3} \Lambda_{\mathrm{cc}}\right)\right)\right.}-1}{\square-\frac{2}{3} \Lambda_{\mathrm{cc}}}, \\
\gamma_{\mathrm{S}}(\square)= & -\frac{1}{6} \frac{e^{H_{\mathrm{S} 2}\left(\tilde{p}\left(\left(\square-\frac{2}{3} \Lambda_{\mathrm{cc}}\right)\left(\square+\frac{4}{3} \Lambda_{\mathrm{cc}}\right)\left(\square-\frac{4}{3} \Lambda_{\mathrm{cc}}\right)\right)\right.}-1}{\square+\frac{4}{3} \Lambda_{\mathrm{cc}}} \\
& -\frac{\square}{12} \frac{e^{\left.H_{\mathrm{S} 2}\left(\tilde{p}\left(\left(\square-\frac{2}{3} \Lambda_{\mathrm{cc}}\right)\left(\square+\frac{4}{3} \Lambda_{\mathrm{cc}}\right)\left(\square-\frac{4}{3} \Lambda_{\mathrm{cc}}\right)\right)\right)\right|_{\square \rightarrow \square+\frac{8}{3} \Lambda_{\mathrm{cc}}}}-1}{\left(\square+2 \Lambda_{\mathrm{cc}}\right)\left(\square+\frac{4}{3} \Lambda_{\mathrm{cc}}\right)} .
\end{aligned}
$$

Therefore, we end up with the form factors already introduced in the footnote above, but with a new polynomial as an argument of the entire function $H_{\mathrm{S} 2}$. Moreover, now $H_{0}=H_{\mathrm{S} 2}$ and the second variation of the action (52) turns into

$$
\begin{aligned}
S_{(\mathrm{A}) \mathrm{dS}}^{(2) \mathrm{CR}}= & \frac{1}{2} \int d^{4} x \sqrt{|\bar{g}|}\left\{\tilde{h}^{\perp \mu \nu}\left(\square-\frac{\bar{R}}{6}\right) e^{H_{\mathrm{S} 2}(\tilde{p})} \tilde{h}_{\mu \nu}^{\perp}\right. \\
& \left.-\tilde{\phi}\left(\square+\frac{\bar{R}}{3}\right) e^{H_{\mathrm{S} 2}(\tilde{p})} \tilde{\phi}\right\} .
\end{aligned}
$$

The second order variation (56) has the same form factor to multiply the tensorial as well as the scalar perturbations. 
Once more we point out that the replacement of $R$ with $\Lambda_{\mathrm{cc}}$ is an off-shell operation just as in the Einstein-Hilbert theory in the presence of a cosmological constant.

\section{QUANTUM FINITENESS}

In this section we study two classes of theories involving, respectively, the Ricci scalar and the off-shell cosmological constant in the form factors. In the first subsection we study the theory (18) with form factors (19), and in the second subsection the theory (18) with form factors (20).

\section{A. Analysis of the theory (18) with form factors}

In agreement with the analysis in the previous section the polynomial appearing in the ultraviolet limit of the form factor can also contain powers of the Ricci scalar, while the nonlocal structure gives contributions only to the finite part of the quantum effective action. Therefore, a quite general polynomial giving a contribution to the beta functions in $D=4$ is

$$
\begin{aligned}
p(z, \mathcal{R})= & a_{\gamma+1}^{(0)} z^{\gamma+1}+a_{\gamma+1}^{(1)} z^{\gamma} \mathcal{R}+a_{\gamma+1}^{(2)} z^{\gamma-1} \mathcal{R}^{2}+\ldots \\
& +a_{\gamma}^{(0)} z^{\gamma}+a_{\gamma}^{(1)} z^{\gamma-1} \mathcal{R}+\ldots+a_{\gamma-1}^{(0)} z^{\gamma-1}
\end{aligned}
$$

For the theories presented in this paper $\mathcal{R}$ can only be the Ricci scalar. The ellipses (...) also include terms arising from commutators of the $\square$ operator with covariant derivatives and curvatures.

To have a finite theory at quantum level (or better conformally invariant) we have to make the beta functions for the following six operators vanish:

$$
\begin{aligned}
& \sqrt{|g|}, \quad \sqrt{|g|} R, \quad \sqrt{|g|} R^{2}, \\
& \sqrt{|g|} \mathbf{R i c}^{2}, \quad \sqrt{|g|} \mathbf{G B}, \quad \sqrt{|g|} \square R,
\end{aligned}
$$

where GB is the Gauss-Bonnet operator. The beta functions $\beta_{R^{2}}, \beta_{R_{\mu \nu}^{2}}$, and $\beta_{\mathbf{G B}}$ [in the basis (58)] get contributions also from the following killers, if they are added to the action:

$$
\begin{aligned}
\mathcal{V}(\mathbf{C})= & s_{w}^{(1)} C_{\mu \nu \rho \sigma} C^{\mu \nu \rho \sigma} \square^{\gamma-2} C_{\alpha \beta \gamma \delta} C^{\alpha \beta \gamma \delta} \\
& +s_{w}^{(2)} C_{\mu \nu \rho \sigma} C^{\alpha \beta \gamma \delta} \square^{\gamma-2} C_{\alpha \beta \gamma \delta} C^{\mu \nu \rho \sigma} .
\end{aligned}
$$

These killers do not spoil the structure of the kinetic operator nor the propagator because the Weyl tensor evaluated on any homogeneous and isotropic spacetime is identically zero and the second order variation on the action based on (59) is at least quadratic in the Weyl tensor. Moreover, they are enough to make zero the two beta functions $\beta_{R^{2}}$ and $\beta_{R_{\mu \nu}^{2}}$. Indeed, the contribution of (59) can be only linear in the front coefficients $s_{w}^{(1)}$ and $s_{w}^{(2)}$ as has been shown in [6] by a direct implementation of the background field method.

If we want to use killers that do not change the structure of the kinetic operator around (A)dS, one option is to build them using only hatted quantities as in footnote 5 (so with the background value of the tensor subtracted, cf. also Appendix). Other viable killers, which possess the same property, are

$s_{s}^{(1)} S_{\mu \nu} S^{\mu \nu} \square^{\gamma-2} S_{\rho \sigma} S^{\rho \sigma}, \quad s_{s}^{(2)} S_{\mu \nu} S^{\rho \sigma} \square^{\gamma-2} S_{\rho \sigma} S^{\mu \nu}$,

where $S_{\mu \nu}$ was defined in (42).

On any MSS background the GB operator is nonvanishing, while $\square R$ always vanishes. Regarding the contributions to the divergent part of the quantum effective action (under an integral) GB and $\square R$ can be neglected as total derivatives on MSS. The reason to kill these two more divergences eventually has to do with the conformal invariance of the theory, but not merely with finiteness.

The beta function for the Newton constant $\beta_{R}$ can be made zero using the following example of the killer operator ${ }^{6}$ :

$$
S_{\mu \nu} S_{\rho}^{\mu} \square{ }^{\gamma-2} S^{\nu \rho} .
$$

Finally, to have a finite theory we need to make the beta function for the cosmological constant vanish. For this achievement we need to explicitly evaluate the divergent contributions to the one-loop effective action that do not contain any curvature tensor. This result was derived for the first time in [37] and also successfully attained by our group [61]. Given the polynomial (57), only the monomials independent of the curvatures can contribute to the $\mathcal{R}^{0}$ divergence. Therefore, the beta function can depend only on the coefficients $a_{\gamma+1}^{(0)}, a_{\gamma}^{(0)}, a_{\gamma-1}^{(0)}$ in (57). For the sake of simplicity we here consider only the theory in Weyl basis (18) with form factors (19). Moreover, we take $\mathrm{H}_{2}=\mathrm{H}_{0}$, but we replace the polynomial (30) with

$$
\begin{aligned}
p_{12} & =\left(\square+\frac{R}{3}\right)^{2}\left(c_{1} \square^{3}+c_{2} \square^{2}+c_{3} \square\right)^{2}\left(\square-\frac{2}{3} R\right)^{4} \\
& =a_{\gamma+1}^{(0)} \square^{12}+a_{\gamma}^{(0)} \square^{11}+a_{\gamma-1}^{(0)} \square^{10}+O(R),
\end{aligned}
$$

and comparing with (57): $a_{\gamma+1}^{(0)}=c_{1}, \quad a_{\gamma}^{(0)}=2 c_{1} c_{2}$, $a_{\gamma-1}^{(0)}=2 c_{1} c_{3}$. Note that with the polynomial (63) we surely

\footnotetext{
${ }^{6}$ Additionally, we can make to vanish the beta functions $\beta_{R^{2}}$, $\beta_{R_{\mu \nu}^{2}}$ and $\beta_{R}$ introducing also the following terms in $\mathcal{V}$,

$$
\begin{aligned}
& s_{r}^{(1)} \hat{R}^{2} \square^{\gamma-2} \hat{R}^{2}, s_{r}^{(2)} \hat{R}_{\mu \nu} \hat{R}^{\mu \nu} \square^{\gamma-2} \hat{R}_{\rho \sigma} \hat{R}^{\rho \sigma} \\
& \text { and } s_{r}^{(3)} \hat{R}_{\mu \nu} \hat{R}^{\mu \nu} \square^{\gamma-2} \hat{R} \text { respectively, } \\
& \text { where } \hat{R}_{\mu \nu}=R_{\mu \nu}-\Lambda_{\mathrm{cc}} g_{\mu \nu} \text { and } \hat{R}=R-4 \Lambda_{\mathrm{cc}} .
\end{aligned}
$$

However, the operators (61) must be used more carefully because the cosmological constant can be present in the beta functions.
} 
avoid the issue of nonlocal counterterms because it is definite positive on the real axis (namely $\sqrt{p_{12}^{2}}=p_{12}$ ). Therefore, $c_{1}, c_{2}$, and $c_{3}$ can be selected to be positive, negative, or zero (at least one of the $c_{i}$ must be nonzero).

The form factors $\gamma_{\mathrm{C}}$ and $\gamma_{\mathrm{S}}$ in the UV are, respectively,

$$
\gamma_{\mathrm{C}} \rightarrow \frac{e^{\gamma_{E} / 2}}{2}\left(\square+\frac{R}{3}\right)^{2}\left(c_{1} \square^{3}+c_{2} \square^{2}+c_{3} \square\right)^{2}\left(\square-\frac{2}{3} R\right)^{4},
$$

$$
\gamma_{\mathrm{S}} \rightarrow-\frac{e^{\gamma_{E} / 2}}{6}\left(\square+\frac{R}{3}\right)\left(c_{1} \square^{3}+c_{2} \square^{2}+c_{3} \square\right)^{2}\left(\square-\frac{2}{3} R\right)^{4} .
$$

Moreover, the operators $O(R)$ do not give a contribution to the beta function for the cosmological constant. Finally, we need to explicitly evaluate the beta function for the cosmological constant $\left(\beta_{\Lambda_{\mathrm{cc}}}\right)$ and select the parameters $a_{\gamma+1}^{(0)}, a_{\gamma}^{(0)}, a_{\gamma-1}^{(0)}$ to make zero $\beta_{\Lambda_{\mathrm{cc}}}$. Once more, the parameters $a_{\gamma+1}^{(0)}, a_{\gamma}^{(0)}, a_{\gamma-1}^{(0)}$ do not run because all of them appear in front of higher derivative operators of a dimension higher than four.

For the theory (18) with form factors (19) we can explicitly show the finiteness of the theory because the beta function $\beta_{\Lambda_{\mathrm{cc}}}$ has been computed in [37,61] for the following prototype theory:

$$
S_{N}=\int d^{4} x \sqrt{|g|}\left(\omega_{N, R} R \square^{N} R+\omega_{N, C} C \square^{N} C\right) .
$$

From the divergent part of the quantum effective action we can read the beta function. The outcome of the computation is [37]

$$
\begin{aligned}
\Gamma_{\mathrm{cc}, \mathrm{div}}^{(1)}= & -\frac{1}{2(4 \pi)^{2}} \frac{1}{\epsilon} \int d^{4} x \sqrt{|g|}\left(\frac{5 \omega_{N-2, C}}{\omega_{N, C}}+\frac{\omega_{N-2, R}}{\omega_{N, R}}\right. \\
& \left.-\frac{5 \omega_{N-1, C}^{2}}{2 \omega_{N, C}^{2}}-\frac{\omega_{N-1, R}^{2}}{2 \omega_{N, R}^{2}}\right) \\
\equiv & -\frac{1}{2 \epsilon} \int d^{4} x \sqrt{|g|} \beta_{\Lambda_{\mathrm{cc}}} .
\end{aligned}
$$

Finally, we have to compare the nonrunning coefficients $\omega_{i, C}$ and $\omega_{i, R}(i=N+1, N, N-1)$, which appear in front of the operators quadratic in the Weyl tensor and in the Ricci scalar in (66), with the parameters in front of the same operators resulting in the action (18) with asymptotic form factors (64) and (65).

Since the issue with UV divergences is probing the UV limit of the theory, this can also be thought of in the following way. The divergences arise because of the coincidence limit of points used as arguments of Green's functions. When points do come closer, the spacetime is effectively flat and they do not see such an effect as the (A)dS curvature radius. That is why all divergences on MSS are the same as on the flat spacetime. Finally, the UV divergences in QFT do not depend on the background, and, therefore, we have background independence of superrenormalizability or finiteness. In other words, if the theory is UV finite around the flat spacetime, then it is also finite around any other background; in particular, this applies to MSS backgrounds.

\section{B. Analysis of the theory (18) with form factors (20)}

We hereby consider the theory (18) with form factors (20). These form factors [and also (47), (48)] depend explicitly on the cosmological constant $\Lambda_{\mathrm{cc}}$ that in general could appear in the beta functions making the search for a finite quantum gravity much more involved. However, it is sufficient to select out polynomials that in the UV regime do not involve the cosmological constant at least in the coefficients $\omega_{i, C}$ and $\omega_{i, R}$ for $i=N+1, N, N-1$. Given the theory (18) with form factors (20) we can select the following asymptotic polynomials:

$$
\begin{aligned}
p_{\mathrm{C}}\left(\square ; \Lambda_{\mathrm{cc}}\right)= & \frac{1}{\Lambda^{8}} \square^{2}\left(\square-\frac{8}{3} \Lambda_{\mathrm{cc}}\right)^{2} \\
& \times\left(\square^{2}+\frac{8}{3} \Lambda_{\mathrm{cc}} \square+\left(\frac{8}{3} \Lambda_{\mathrm{cc}}\right)^{2}\right), \\
p_{\mathrm{S}}\left(\square ; \Lambda_{\mathrm{cc}}\right)= & \frac{1}{\Lambda^{8}} \square^{2}\left(\square+\frac{4}{3} \Lambda_{\mathrm{cc}}\right)^{2} \\
& \times\left(\square^{2}-\frac{4}{3} \Lambda_{\mathrm{cc}} \square+\left(\frac{4}{3} \Lambda_{\mathrm{cc}}\right)^{2}\right) .
\end{aligned}
$$

Notice that the two parabolic trinomials on the right sides in (68) and (69) are positive for any value of $\square$ and $\Lambda_{\mathrm{cc}}>0$. For the above selected polynomials (68) and (69), $\omega_{N-1, C(R)}=0$ and $\omega_{N-2, C(R)}=0$. Indeed,

$$
\begin{aligned}
\gamma_{\mathrm{C}}(\square)= & \frac{1}{2} \frac{\left(e^{H_{2}\left(\square-\frac{8}{3} \Lambda_{\mathrm{cc}}\right)}-1\right)}{\square-\frac{8}{3} \Lambda_{\mathrm{cc}}} \rightarrow \frac{1}{2} \frac{1}{\Lambda^{8}} \square^{2}\left(\square-\frac{8}{3} \Lambda_{\mathrm{cc}}\right) \\
& \times\left(\square^{2}+\frac{8}{3} \Lambda_{\mathrm{cc}} \square+\left(\frac{8}{3} \Lambda_{\mathrm{cc}}\right)^{2}\right) \\
= & \frac{1}{2 \Lambda^{8}}\left(\square^{5}-\frac{512 \Lambda_{\mathrm{cc}}^{3} \square^{2}}{27}\right), \\
\gamma_{\mathrm{S}}(\square)= & -\frac{1}{6} \frac{\left(e^{H_{0}\left(\square, \Lambda_{\mathrm{cc}}\right)}-1\right)}{\square+\frac{4}{3} \Lambda_{\mathrm{cc}}} \rightarrow-\frac{1}{6} \frac{1}{\Lambda^{8}} \square^{2}\left(\square+\frac{4}{3} \Lambda_{\mathrm{cc}}\right) \\
& \times\left(\square^{2}-\frac{4}{3} \Lambda_{\mathrm{cc}} \square+\left(\frac{4}{3} \Lambda_{\mathrm{cc}}\right)^{2}\right) \\
= & -\frac{1}{6 \Lambda^{8}}\left(\square^{5}+\frac{64 \Lambda_{\mathrm{cc}}^{3} \square}{27}\right) .
\end{aligned}
$$


Therefore, there is no contribution to the beta functions $\beta_{\Lambda_{\mathrm{cc}}}$ and $\beta_{\kappa}$. More importantly, the cosmological constant does not appear in any beta function. In general, we only need the beta function for the cosmological constant to be independent of $\Lambda_{\mathrm{cc}}$ itself to achieve one-loop exact superrenormalizability or finiteness because $\kappa_{4}$ does not appear in the form factors and, therefore, in the beta functions. Let us expand a little on this point. If the beta functions do not depend on any of the running couplings, then we can make them zero at any energy scale and at any loop order by adding suitably selected killer operators because the superrenormalizability implies that the beta functions are oneloop exact.

\section{FIELD REDEFINITION AND TREE-LEVEL PERTURBATIVE TRIVIALITY}

In this section we explicitly show that for a large class of theories involving neither the Riemann nor the Weyl tensor, a field redefinition theorem provides an explanation for the stability of MSS in weakly nonlocal theories. Namely all these theories are tree-level equivalent to the EinsteinHilbert theory in the presence of the cosmological constant. Let us consider the theory (41) with $\gamma_{\mathrm{C}}=0$, namely

$$
\begin{aligned}
S_{\mathrm{NL}-\Lambda}= & -2 \kappa_{4}^{-2} \int d^{4} x \sqrt{|g|}\left[R-2 \Lambda_{\mathrm{cc}}+\mathbf{S} \gamma_{\mathrm{S} 2}(\square) \mathbf{S}\right. \\
& \left.+R \gamma_{\mathrm{S}}(\square) R+\mathcal{V}(\square, \mathbf{R i c}, R)\right] .
\end{aligned}
$$

We can now recast the above action in a way that explicitly shows Einstein's gravitational EOM in the presence of a cosmological constant, i.e.,

$$
\begin{array}{ll}
E_{\mu \nu}=G_{\mu \nu}+\Lambda_{\mathrm{cc}} g_{\mu \nu}, & R_{\mu \nu}=E_{\mu \nu}-\frac{1}{2} g_{\mu \nu} E_{\alpha}^{\alpha}+\Lambda_{\mathrm{cc}} g_{\mu \nu}, \\
S_{\mu \nu}=E_{\mu \nu}-\frac{1}{4} g_{\mu \nu} E_{\alpha}^{\alpha}, & R=-E_{\alpha}^{\alpha}+4 \Lambda_{\mathrm{cc}} .
\end{array}
$$

Making use of the EOM (71), the action now equivalently turns into

$$
\begin{aligned}
S_{\mathrm{NL}-\Lambda}= & -2 \kappa_{4}^{-2} \int d^{4} x \sqrt{|g|}\left[R-2 \Lambda_{\mathrm{cc}}\right. \\
& +\left(E_{\mu \nu}-\frac{1}{4} E g_{\mu \nu}\right) \gamma_{\mathrm{S} 2}\left(\square, E, \Lambda_{\mathrm{cc}}\right)\left(E^{\mu \nu}-\frac{1}{4} E g^{\mu \nu}\right) \\
& +\left(E-4 \Lambda_{\mathrm{cc}}\right) \gamma_{\mathrm{S}}\left(\square, E, \Lambda_{\mathrm{cc}}\right)\left(E-4 \Lambda_{\mathrm{cc}}\right) \\
& \left.+\mathcal{V}\left(\square, \mathbf{E}, E, \Lambda_{\mathrm{cc}}\right)\right],
\end{aligned}
$$

where $\mathbf{E}$ stays for $E_{\mu \nu}$ and $E \equiv E_{\mu}^{\mu}$. The nonlocal form factor $\gamma_{\mathrm{S}}$ satisfies the property $\gamma_{\mathrm{S}}(0)=0$ [see, for example, (55) with the polynomial (53)]. Therefore, we can rewrite the action in the following simplified form:

$$
\begin{aligned}
S_{\mathrm{NL}-\Lambda}= & -2 \kappa_{4}^{-2} \int d^{4} x \sqrt{|g|}\left[R-2 \Lambda_{\mathrm{cc}}+E_{\mu \nu} F^{\mu \nu, \rho \sigma} E_{\rho \sigma}\right], \\
F^{\mu \nu, \rho \sigma} \equiv & \gamma_{\mathrm{S} 2}\left(\square, E, \Lambda_{\mathrm{cc}}\right)\left(g^{\mu \rho} g^{\nu \sigma}-\frac{1}{4} g^{\mu \nu} g^{\rho \sigma}\right) \\
& +\gamma_{\mathrm{S}}\left(\square, E, \Lambda_{\mathrm{cc}}\right) g^{\mu \nu} g^{\rho \sigma}+\tilde{\mathcal{V}}^{\mu \nu \rho \sigma}\left(\square, \mathbf{E}, E, \Lambda_{\mathrm{cc}}\right),
\end{aligned}
$$

where the potential $\mathcal{V}\left(\square, \mathbf{E}, E, \Lambda_{\mathrm{cc}}\right)$ must be at least quadratic in the EOM $E_{\mu \nu}$, namely

$$
\mathcal{V}\left(\square, \mathbf{E}, E, \Lambda_{\mathrm{cc}}\right)=E_{\mu \nu} \tilde{\mathcal{V}}^{\mu \nu \rho \sigma}\left(\square, \mathbf{E}, E, \Lambda_{\mathrm{cc}}\right) E_{\rho \sigma} .
$$

In the view of the restructured action (73), we are now ready to implement the following general theorem in the presence of a cosmological constant. An analogous theorem was previously proved and applied to the case without a cosmological constant [14].

Theorem: By making use of a proper analytic field redefinition $g \rightarrow g^{\prime}$ the action (73) can be recast in the Einstein-Hilbert form with the presence of a cosmological constant term, i.e.,

$$
\mathcal{L}_{\mathrm{EH}-\Lambda}=-2 \kappa_{4}^{-2} \sqrt{|g|}\left(R-2 \Lambda_{\mathrm{cc}}\right),
$$

provided that $\mathcal{V}$ has the structure given in (75); namely it is at least quadratic in $E_{\mu \nu}$ and/or $E_{\alpha}^{\alpha}$ and does not contain any Riemann or Weyl tensor explicitly. Therefore, the theorem does not apply to the theory with $\gamma_{C} \neq 0$.

Proof: The proof is based on a perturbative field redefinition $g \rightarrow g^{\prime}$ to all orders in the Taylor expansion with respect to the redefinition of the metric field. First, we assume that we have given two general weakly nonlocal action functionals $S^{\prime}(g)$ and $S\left(g^{\prime}\right)$, respectively defined in terms of the metric fields $g$ and $g^{\prime}$, such that

$$
S^{\prime}(g)=S(g)+E_{i}(g) F_{i j}(g) E_{j}(g),
$$

where $F$ can contain derivative operators and $E_{i}=\delta S / \delta g_{i}$ are the EOM of the theory with the action $S(g) .^{7}$ The statement of the theorem is that there exists a field redefinition

$$
g_{i}^{\prime}=g_{i}+\Delta_{i j} E_{j}, \quad \Delta_{i j}=\Delta_{j i},
$$

\footnotetext{
${ }^{7}$ Here we use a compact deWitt notation, and with the indices $i, j$ on fields we encode all Lorentz, group indices, and the spacetime dependence of the fields. Additionally, we assume that the field space is flat and we do not need to raise indices in sums there.
} 
such that, perturbatively in $F$, but to all orders in powers of $F$ in the field redefinition $g \rightarrow g^{\prime},{ }^{8}$ we have the equivalence

$$
S^{\prime}(g)=S\left(g^{\prime}\right)
$$

Above $\Delta_{i j}$ is a possibly nonlocal operator acting linearly on the EOM $E_{j}$, with indices $i$ and $j$ in the field space, and it is defined perturbatively in powers of the operator $F_{i j}(g)$, namely $\Delta_{i j}=F_{i j}(g)+\ldots$. Let us consider the first order in Taylor expansion for the functional $S\left(g^{\prime}\right)$, which reads

$$
S\left(g^{\prime}\right)=S(g+\delta g) \approx S(g)+\frac{\delta S}{\delta g_{i}} \delta g_{i}=S(g)+E_{i} \delta g_{i} .
$$

If we can find a weakly nonlocal expression for $\delta g_{i}$ such that $S^{\prime}(g)=S(g)+E_{i} \delta g_{i}$ (note that the argument of the functionals $S^{\prime}$ and $S$ is now the same), then there exists a field redefinition $g \rightarrow g^{\prime}$ satisfying (79). Hence the two actions $S^{\prime}(g)$ and $S\left(g^{\prime}\right)$ are tree-level equivalent.

As is obvious from above, in the proof of our theorem it was crucial to use the classical EOM $E_{i}$. In the theory (73) this implies $\mathbf{E}=0$ (here no matter source is present).

Now we can explicitly apply the above field redefinition theorem to our class of theories (73), where we do not include terms with the Riemann tensor $R_{\mu \nu \rho \sigma}$ nor the Weyl tensor $C_{\mu \nu \rho \sigma}$ in the action. Since we are interested in $S\left(g^{\prime}\right) \equiv S_{\mathrm{EH}-\Lambda}\left(g^{\prime}\right)$ and $S^{\prime}(g) \equiv S_{\mathrm{NL}-\Lambda}(g)$, the relation (77) reads

$$
\begin{aligned}
S\left(g^{\prime}\right) & =S_{\mathrm{EH}-\Lambda}(g)-2 \kappa_{4}^{-2} \int d^{4} x \sqrt{|g|} E_{\mu \nu}(g) F^{\mu \nu, \rho \sigma}(g) E_{\rho \sigma}(g) \\
& =S^{\prime}(g),
\end{aligned}
$$

where $E_{\mu \nu}$ is given in (71), $F^{\mu \nu, \rho \sigma}(g)$ is defined in (74), and $\mathcal{V}$ compatible with the field redefinition has been introduced in (75).

As a particular implication of the theorem we can always make a field redefinition to turn the kinetic operator and the propagator for the gravitational fluctuations of the nonlocal theory into the one of Einstein's gravity plus the cosmological constant. Moreover, when we can properly define asymptotic graviton states in a MSS, all the tree-level onshell $n$-point functions for the weakly nonlocal theory (73) are exactly the same as the ones for the Einstein-Hilbert- $\Lambda_{\mathrm{cc}}$ gravity (76).

\footnotetext{
${ }^{8}$ The field redefinition preserves general covariance. Indeed, formula (77) shows a multiple product of weakly nonlocal factors $F_{i j}$ and the EOM $E_{i}$, which are both covariant under active general coordinate transformations (diffeomorphisms). Additionally, the asymptotic behavior of the field redefinitions is such that they go to zero sufficiently fast at infinity together with the fast falloff of fields in order to preserve the spectrum of the theory. As a corollary, the large diffeomorphisms, are not touched by such field redefinitions.
}

Finally, in view of the theorem proved here, it is clear why at tree level a class of weakly nonlocal theories and the local Einstein-Hilbert theory with the cosmological constant have the same spectrum and the same $n$-point functions; ergo this range of weakly nonlocal theories is actually local at a classical perturbative level. However, we cannot push further the outcome of the theorem because in a theory with an infinite number of derivatives at the moment we do not know the number of nonperturbative d.o.f., in contrast to the Einstein-Hilbert theory where the ADM formulation ensures that there are only 2 d.o.f. at perturbative and nonperturbative levels and around any background. Similarly this theorem likely does not hold at quantum level.

To summarize the content of this section, we proved that the Einstein-Hilbert- $\Lambda_{\mathrm{cc}}$ theory (EH- $\Lambda$ ) and nonlocal gravity with the presence of a cosmological constant term are equivalent at the perturbative level. The proof is based on a field redefinition theorem that works perturbatively in $F_{i j}$, but to all perturbative orders in $\Delta_{i j}$. The above result can therefore be seen as a resummation of all perturbative contributions. In the previous sections we have proved that the EOM for both the theories, EH- $\Lambda$ and nonlocal gravity, have the same solutions at the linear order in the gravitational perturbation, and, therefore, we inferred that the two theories have the same perturbative spectrum. The theorem in this section guarantees that the classical $n$-point functions (if they can be defined in AdS and/or dS spacetimes) are also the same in the two theories. The theorem is particularly useful in Minkowski spacetime where the $n$-point functions are surely well defined. On the other hand, we do not know if the spectrum of the two theories still coincides at the nonperturbative level and/or on a general background. Actually, on a general background we expect more d.o.f. in nonlocal gravity contrary to what happens in the EH- $\Lambda$ theory as recently proved in $[62,63]$.

At quantum level the two theories are completely different: the EH- $\Lambda$ theory is nonrenormalizable, while nonlocal gravity is finite. Indeed, the field redefinition surely changes the measure in the path integral, and the two theories show different behaviors at quantum level. We could say that the field redefinition is anomalous because at quantum level other finite operators can be generated, as, for example, Rieman ${ }^{3}$, and then the mapping between $\mathrm{EH}-\Lambda$ and nonlocal gravity does not work anymore. (In the proof we assumed the action to be quadratic in the EOM, but the finite quantum corrections can violate such an assumption.)

\section{MORE ON PROPAGATORS IN WEAKLY NONLOCAL THEORIES}

In this section we are going to extend our own construction of propagators in weakly nonlocal theories. Let us consider a simple example of a weakly nonlocal scalar field theory and its propagator, namely 
$S=\int d^{D} x \varphi f(\square)\left(\square+m^{2}\right) \varphi \Rightarrow \Pi=\frac{1}{f(\square)\left(\square+m^{2}\right)}$.

The above theory in most cases comes as a generalization of a local second order theory whose action and propagator, respectively, read

$$
S=\int d^{D} x \varphi\left(\square+m^{2}\right) \varphi \Rightarrow \Pi=\frac{1}{\square+m^{2}} .
$$

One of the most frequent situations is the wish to constrain $f(\square)$ such that both theories have the same physical excitations. In this example this means that each theory describes only a single scalar, the mathematical requirement for this is that $f(\square)$ has no zeros, and hence the propagator in (82) has no extra new poles on the whole complex plane besides the one at $-m^{2}$. That is, $f(z) \neq 0$ for all $|z|<\infty, z \in \mathbb{C}$, where $z=\square$.

The usual way used to achieve no extra poles in the propagator in (82) is to require

$$
f(z)=e^{\alpha(z)} \quad \text { where } \alpha(z) \text { is an entire function. }
$$

This indeed works and propagates from [12] through all the papers known to us on the subject of weakly nonlocal theories. By definition an entire function is a function analytic on the whole complex plane. As such it has no poles in any finite region of the complex plane. The exponent of any finite (and zero) argument is always a nonzero complex number. Actually, the exponent of an entire function is a special entire function with no zeros on the whole complex plane. As a result $f(z)$ in (84) is always nonzero. Moreover, a particular setup may be required to preserve the normalization of the propagator in the lowenergy limit. This implies $f(0)=1$ or equivalently $\alpha(0)=0$. Physically this can be understood as follows: given there is a scale $\Lambda$ which defines the characteristic scale of $f(\square)$ such that this function is truly $f\left(\square / \Lambda^{2}\right)$, one may want to see the modified model (82) returning to its local counterpart (83) when $\Lambda \rightarrow \infty$. The latter is the local theory limit.

At this point we put the following claim.

Claim: The form of $f(\square)$ given by (84) is overrestricted and is not necessary as long as the number of d.o.f. is concerned. Instead we can prove the following proposition.

Proposition: The less restrictive form that is still compatible with the requirements (i) to avoid a generation of new d.o.f., (ii) to keep the original normalization in the local limit, and (iii) to preserve and/or improve the UV behavior of the propagator is

$f(z)=\frac{e^{\alpha(z)}}{\beta(z)}$ where $\alpha(z), \quad \beta(z)$ are entire functions.
Indeed, substituting this in the propagator in (82) one gets

$$
\Pi=\frac{\beta(\square)}{e^{\alpha(\square)}\left(\square+m^{2}\right)} .
$$

The exponent in the denominator works exactly as it worked before when the form (84) was used. The crucial thing to understand is that the new function $\beta(\square)$ does not change either of required properties (i)-(iii) as long as it is an entire function. This is a trivial consequence of the definition of an entire function that says it has no poles on the whole complex plane and, as such, our propagator has no new poles as well. The normalization in the local limit can always be preserved by the demand $\beta(0) \exp (-\alpha(0))=1$. The UV behavior is subject to a particular choice of the functions $\alpha(\square)$ and $\beta(\square)$, which are almost unrestricted so far in any way.

A point of worry is instead the EOM, which we are going to consider in more detail. The EOM can be written as

$$
\frac{e^{\alpha(\square)}\left(\square+m^{2}\right)}{\beta(\square)} \varphi=0 .
$$

We start with reminding the reader that thanks to the Weierstrass factorization theorem [64] any entire function $\beta(z)$ can be represented as

$$
\beta(z)=e^{\tilde{\beta}(z)} \prod_{I}\left(z-z_{I}\right)^{m_{I}},
$$

where $\tilde{\beta}(z)$ is again an entire function, $z_{I}$ are roots of $\beta(z)$, and $m_{I}$ are their multiplicities. First of all, we stress that $\beta(z)$ in the condition of the theorem is an entire function and as such in general the $1 / \beta(z)$ factor in EOM (87) cannot be presented like this. Consequently, and not surprisingly, we do not gain new factors in the numerator of EOM. Having $\alpha(z)$ and $\tilde{\beta}(z)$ both entire functions we can join them into a redefined function $\tilde{\alpha}(z)=\alpha(z)-\tilde{\beta}(z)$. So, without any assumptions we can write the EOM (87) as

$$
\frac{e^{\tilde{\alpha}(\square)}\left(\square+m^{2}\right)}{\prod_{I}\left(\square-z_{I}\right)^{m_{I}}} \varphi=0 .
$$

We assume that by construction neither of $z_{I}$ coincides with $-m^{2}$. Otherwise, we would immediately write another EOM and propagator. Then a canonical solution originates from the mode

$$
\left(\square+m^{2}\right) \varphi=0
$$


Further, it was shown in [65] that the exponent operator does not generate new solutions and we can drop it from the consideration of solutions of the EOM. A simple way to see that the denominator does not provide new solutions, which could be associated with new d.o.f., is to notice that we can use the Schwinger and Feynman parametrization to achieve

$$
\begin{aligned}
& \frac{1}{\prod_{I}\left(\square-z_{I}\right)^{m_{I}}} \varphi \\
& =\frac{\Gamma\left(\sum_{I} m_{I}\right)}{\prod_{I} \Gamma\left(m_{I}\right)} \int_{0}^{1}\left(\prod_{I} d u_{I}\right) \frac{\delta\left(1-\sum_{I} u_{I}\right) \prod_{I} u_{I}^{m_{I}-1}}{\left[\sum_{I} u_{I}\left(\square-z_{I}\right)\right]^{\sum_{I} m_{I}}} \varphi \\
& =\frac{1}{\prod_{I} \Gamma\left(m_{I}\right)} \int_{0}^{1}\left(\prod_{I} d u_{I} u_{I}^{m_{I}-1}\right) \\
& \quad \times \delta\left(1-\sum_{I} u_{I}\right) \int_{0}^{\infty} d s s \sum_{I}^{m_{I}-1} e^{s \sum_{I} u_{I} z_{I}} e^{-s \sum_{I} u_{I} \square} \varphi .
\end{aligned}
$$

This is again an exponential of the d'Alembertian operator acting on the scalar field $\varphi$. Therefore, we can say that no new solutions are generated as long as we can change the order of differentiation and integration. The latter is true as long as a Laplace transform of the scalar field function can be defined. The classical field in turn has to have a well defined Laplace transform in order to be properly quantized.

The situation becomes trickier when we have to define and solve for the Green function that is defined as a solution to the fundamental equation

$$
\frac{e^{\tilde{\alpha}(\square)}\left(\square+m^{2}\right)}{\prod_{I}\left(\square-z_{I}\right)^{m_{I}}} G\left(x, x^{\prime}\right)=\delta\left(x-x^{\prime}\right),
$$

with appropriate boundary conditions (retarded, advanced, causal, etc.). Here we can act in analogy with the treatment of the $1 / \square$ operator in gravity theories as in [66]. However, a consistent treatment exists for the single inverse d'Alembertian only. We do not need Green functions of this (or any other) kind to proceed, but we very much hope to see this question solved in future works.

Two more comments are in order here. First, the new form (85) is definitely a significant extension of the class of possible form factors that can enter in weakly nonlocal theories. Second, it will be shown below that such an extension is crucial to guarantee the no-ghost conditions in both regimes: quantum gravity and inflation.

For the case of gravitational theories the propagator (86), especially the new higher derivative factors must obey several conditions that were first formulated in $[1,3]$ and are given above in Sec. II.
These conditions are aimed at achieving the maximum convergence of loop integrals still preserving the power law falloff of the integrands at infinity. The latter is important to preserve the locality of counterterms and as such to maintain the renormalizability of the theory [5,6,31]. Prior to the current analysis the conditions in question were considered for the function $f(z)$ as it is given in (84). However, it is easy to see that no extra complications arise when we have to satisfy the above conditions using the function $f(z)$ in (85) in kinetic operators for theory not involving gravity or any other non-Abelian gauge theory.

Going further one can easily understand that the form (85) is again not an ultimate nonlocal factor. That factor was constructed under the assumption that we do not alter the already existing pole at $\square=-m^{2}$ in the propagator in (82). Under this assumption the nonlocal factor is still maximally general. However, this requirement can in principle be relaxed unless we have some external reasons to maintain this property. Having said this, we understand that we can suggest a function

$f(z)=\frac{e^{\alpha(z)}}{\beta(z)} \frac{z+\mu^{2}}{z+m^{2}} \quad$ where $\alpha(z), \beta(z)$ are entire functions,

which being substituted in the propagator (82) results in

$$
\Pi=\frac{\beta(\square)}{e^{\alpha(\square)}\left(\square+\mu^{2}\right)} .
$$

This clearly propagates only a scalar with a new mass square $\mu^{2}$ while all other properties remain the same. From here actually no further generalization is seen as long as we preserve the number of poles. The latter property is indeed very important because new poles will be ghosts due to the Ostrogradsky instability [67].

In an extreme case we can have a very special function

$$
\begin{aligned}
f(z)= & \frac{e^{\alpha(z)}}{\beta(z)} \frac{1}{z+m^{2}} \quad \text { where } \alpha(z) \\
& \text { and } \beta(z) \text { are entire functions, }
\end{aligned}
$$

which being substituted in the propagator (82) results in

$$
\Pi=\frac{\beta(\square)}{e^{\alpha(\square)}} .
$$

This is a clear analog of a Lagrangian of a $p$-adic theory which has no poles and as such no propagating d.o.f. in the perturbative vacuum at all. 
The following comment follows. Our generalized construction is transparent and obviously valid as long as everything but gravity is concerned. As an immediate example, what we have just discussed helps in understanding the behavior of quantum perturbations during inflation as will be explained in details in [44]. This is because the propagator for perturbations explicitly and generically has a numerator factor which is $\beta(\square)$ above. Concerning gravity, the propagator (96) comes together with strongly nonlocal vertices. The role of these new vertices in the perturbative unitarity is at the moment not under control and deserves much more investigation.

\section{CONCLUSIONS}

In this paper we explicitly proved that all the weakly nonlocal gravitational theories consistent at quantum level have exactly the same classical properties as Einstein's gravity at linear level when studied perturbatively around any maximally symmetric spacetime background. These theories differ only for the presence or not of the Weyl tensor in the nonlocal operators quadratic in curvatures, but the outcome is always the same. Namely, the quadratic action at the second order in the graviton perturbations around any MSS can be recast in the form of the Einstein-Hilbert quadratized action up to exponential form factors in front of the corresponding projectors for the spin-two and spin-zero components. For one out of the two ranges of theories, namely the one without a Weyl or Riemann tensor in the action, we proved, making use of a field redefinition theorem, that the theory is perturbatively (in the entire function defining the field redefinition) equivalent to the Einstein-Hilbert action in the presence of a cosmological constant. This statement holds to all orders in Taylor expansion in the field redefinition of the metric tensor. Moreover, the field redefinition theorem, when the graviton's asymptotic states on a MSS are properly defined, endorses that all tree-level $n$-point scattering amplitudes in the weakly nonlocal theory coincide with the ones of Einstein-Hilbert gravity with a cosmological constant on the same MSS background.

At quantum level, for one out of the two classes of theories (namely the one in Weyl's basis) we explicitly proved that all the beta functions can be made to vanish. Therefore, the quantum theory is finite (in the DIMREG scheme) on any MSS. Certainly, the theory in the Ricci basis also enjoys the same convergence properties.
We can finally claim that the weakly nonlocal theories are perturbatively well defined, unitary (as long as the Einstein-Hilbert is), and finite at quantum level on any maximally symmetric space. Having an ultraviolet complete theory for gravity in the quantum field theory framework, we can now study the implications and/or applications in the AdS/CFT domain. The AdS/CFT correspondence is clearly defined, but there is no clear definition of the dS/CFT correspondence, unless one appeals to a nonlocal map between the AdS and dS spaces as discussed in [68]. However, we remark that our construction is valid and works equally well for both signs of the cosmological constant. First, preliminary results show that the transition from dS to AdS can be reached as an effect of RG flow of the couplings of the theory. Second, of course, we understand that for holding gauge/gravity duality even simple kinematical conditions must be satisfied (such as the equivalence between the group of isometries of $\mathrm{AdS}$ and the conformal group in flat Minkowski spacetime), and these are not true on dS. However, here we treat dS/AdS as backgrounds and for the moment quantum backreaction is neglected. We may express a belief that including backreaction effects in a particular class of theories studied in this paper will show some preference toward the sign of the curvature of the background, and then we could ultimately decide whether we can or why we cannot extend the duality to a dS spacetime. We are interested to export all the results obtained in string theory and in the AdS/CFT correspondence to nonlocal quantum gravity, and we will surely invest time and resources on this topic in the future. We believe it will be interesting to see whether nonlocal gravity could shed light on various conceptual problems associated with possible $\mathrm{dS} / \mathrm{CFT}$ correspondence.

\section{ACKNOWLEDGMENTS}

A. K. is supported by FCT Portugal investigator Project No. IF/01607/2015, FCT Portugal fellowship SFRH/BPD/ 105212/2014, and in part by FCT Portugal Grant No. UID/ MAT/00212/2013.

\section{APPENDIX: VARIATIONS}

Here we collect the results about variations on a maximally symmetric background of operators quadratic in curvature. We write them in a manifestly self-adjoint form. First, the variation of action written with Weyl tensors reads 


$$
\begin{aligned}
\frac{1}{2} \delta^{2}\left(\int d^{D} x \sqrt{|g|} C_{\mu \nu \rho \sigma} \mathcal{F}(\square) C^{\mu \nu \rho \sigma}\right) \\
\overline{\overline{\mathrm{MSS}}}=\int d^{d} x \sqrt{|g|}\left[h_{\mu \nu}\left(\frac{2 D(D-3)}{(D-2)(D-1)^{2}} \Lambda_{\mathrm{cc}}^{2}-\frac{(D-3)(D+2)}{(D-2)(D-1)} \Lambda_{\mathrm{cc}} \square+\frac{D-3}{D-2} \square^{2}\right) \mathcal{F}\left(\square+2 \frac{D-2}{D-1} \Lambda_{\mathrm{cc}}\right) h^{\mu \nu}\right. \\
\quad+h\left[\left(-\frac{2(D-3)}{(D-2)(D-1)^{2}} \Lambda_{\mathrm{cc}}^{2}+\frac{(D-3)(D+2)}{D(D-2)(D-1)} \Lambda_{\mathrm{cc}} \square-\frac{D-3}{D(D-2)} \square^{2}\right) \mathcal{F}\left(\square+2 \frac{D-2}{D-1} \Lambda_{\mathrm{cc}}\right)\right. \\
\left.\quad-\left(\frac{2(D-3)}{D(D-2)(D-1)} \Lambda_{\mathrm{cc}} \square+\frac{D-3}{D(D-2)(D-1)} \square^{2}\right) \mathcal{F}\left(\square+4 \Lambda_{\mathrm{cc}}\right)\right] h \\
\quad+h_{\mu \nu} \nabla^{\mu} \nabla^{\nu}\left(\frac{2(D-3)}{(D-2)(D-1)} \Lambda_{\mathrm{cc}}+\frac{D-3}{(D-2)(D-1)} \square\right) \mathcal{F}\left(\square+4 \Lambda_{\mathrm{cc}}\right) h \\
\quad+h\left(\frac{2(D-3)}{(D-2)(D-1)} \Lambda_{\mathrm{cc}}+\frac{D-3}{(D-2)(D-1)} \square\right) \mathcal{F}\left(\square+4 \Lambda_{\mathrm{cc}}\right) \nabla_{\mu} \nabla_{\nu} h^{\mu \nu} \\
\quad+h_{\mu \nu} \nabla^{\mu}\left(-\frac{2(D-3)}{(D-2)(D-1)} \Lambda_{\mathrm{cc}}-\frac{2(D-3)}{D-2} \square\right) \mathcal{F}\left(\square+3 \Lambda_{\mathrm{cc}}\right) \nabla_{\rho} h^{\nu \rho} \\
\left.\quad+\frac{D-3}{D-1} h_{\mu \nu} \nabla^{\mu} \nabla^{\nu} \mathcal{F}\left(\square+4 \Lambda_{\mathrm{cc}}\right) \nabla_{\rho} \nabla_{\sigma} h^{\rho \sigma}\right] .
\end{aligned}
$$

Next we introduce the definition $\hat{R}=R-D \Lambda_{\mathrm{cc}}=R-\bar{R}$, and we evaluate the following variation:

$$
\begin{aligned}
& \frac{1}{2} \delta^{2}\left(\int d^{D} x \sqrt{|g|} \hat{R} \mathcal{F}(\square) \hat{R}\right) \\
& \overline{\overline{\mathrm{MSS}}} \int d^{D} x \sqrt{|g|}\left[h\left(\Lambda_{\mathrm{cc}}+\square\right)^{2} \mathcal{F}(\square) h\right. \\
& \quad-h_{\mu \nu} \nabla^{\mu} \nabla^{\nu}\left(\Lambda_{\mathrm{cc}}+\square\right) \mathcal{F}(\square) h \\
& \quad-h\left(\Lambda_{\mathrm{cc}}+\square\right) \mathcal{F}(\square) \nabla_{\mu} \nabla_{\nu} h^{\mu \nu} \\
& \left.\quad+h_{\mu \nu} \nabla^{\mu} \nabla^{\nu} \mathcal{F}(\square) \nabla_{\rho} \nabla_{\sigma} h^{\rho \sigma}\right]
\end{aligned}
$$

On a MSS we have the following values of the background curvatures:

$\bar{R}_{\mu \nu \rho \sigma}=\frac{2 \Lambda_{\mathrm{cc}}}{D-1} g_{\mu[\rho} g_{\sigma] \nu}, \quad \bar{R}_{\mu \nu}=\Lambda_{\mathrm{cc}} g_{\mu \nu}, \quad \bar{R}=D \Lambda_{\mathrm{cc}}$.

An useful formula is

$\hat{R} \mathcal{F}(\square) \hat{R}=R \mathcal{F}(\square) R-2 D \mathcal{F}_{0} \Lambda_{\mathrm{cc}} R+D^{2} \mathcal{F}_{0} \Lambda_{\mathrm{cc}}^{2}$, where $\mathcal{F}_{0}=\mathcal{F}(0)$.

$$
\begin{aligned}
& \frac{1}{2} \delta^{2}\left(\int d^{D} x \sqrt{|g|} a_{\Lambda}\right)=\int d^{D} x \sqrt{|g|}\left(\frac{1}{8} h^{2}-\frac{1}{4} h_{\mu \nu} h^{\mu \nu}\right) a_{\Lambda}, \\
& \text { (A5) } \\
& \frac{1}{2} \delta^{2}\left(\int d^{D} x \sqrt{|g|} R\right) \\
& \underset{\mathrm{MSS}}{=} \int d^{D} x \sqrt{|g|}\left[\frac{1}{4} h_{\mu \nu} \square h^{\mu \nu}-\frac{1}{4} h \square h+\frac{1}{4} h \nabla_{\mu} \nabla_{\nu} h^{\mu \nu}\right. \\
& \quad+\frac{1}{4} h_{\mu \nu} \nabla^{\mu} \nabla^{\nu} h-\frac{1}{2} h_{\mu_{\nu}} \nabla^{\mu} \nabla_{\rho} h^{\nu \rho} \\
& \left.\quad+\Lambda_{\mathrm{cc}}\left(-\frac{D^{2}-3 D+4}{4(D-1)} h_{\mu \nu} h^{\mu \nu}+\frac{D^{2}-5 D+8}{8(D-1)} h^{2}\right)\right] .
\end{aligned}
$$

The variations of the cosmological constant $a_{\Lambda}$ and the

For completeness we give also the expression for the variation (in normal form) in $D=4$,

$$
\begin{aligned}
\frac{1}{2} \delta^{2}\left(\int d^{4} x \sqrt{|g|} C_{\mu \nu \rho \sigma} \mathcal{F}(\square) C^{\mu \nu \rho \sigma}\right)_{\mathrm{MSS}} & \int d^{4} x \sqrt{|g|}\left[h_{\mu \nu}\left(\frac{4}{9} \Lambda_{\mathrm{cc}}^{2}-\Lambda_{\mathrm{cc}} \square+\frac{1}{2} \square^{2}\right) \mathcal{F}_{2} h^{\mu \nu}+h\left(-\frac{1}{9} \Lambda_{\mathrm{cc}}^{2}+\frac{5}{18} \Lambda_{\mathrm{cc}} \square-\frac{1}{6} \square^{2}\right) \mathcal{F}_{2} h\right. \\
& +h_{\mu \nu} \nabla^{\mu} \nabla^{\nu}\left(-\frac{1}{3} \Lambda_{\mathrm{cc}}+\frac{1}{6} \square\right) \mathcal{F}_{2} h+h \nabla_{\mu} \nabla_{\nu}\left(-\frac{1}{9} \Lambda_{\mathrm{cc}}+\frac{1}{6} \square\right) \mathcal{F}_{2} h^{\mu \nu} \\
& \left.+h_{\mu \nu} \nabla^{\mu} \nabla_{\rho}\left(\frac{4}{3} \Lambda_{\mathrm{cc}}-\square\right) \mathcal{F}_{2} h^{\nu \rho}+\frac{1}{3} h_{\mu \nu} \nabla^{\mu} \nabla^{\nu} \nabla_{\rho} \nabla_{\sigma} \mathcal{F}_{2} h^{\rho \sigma}\right]
\end{aligned}
$$


where we introduced a short notation for the form factor with a translated argument,

$$
\mathcal{F}_{2} \equiv \mathcal{F}\left(\square+\frac{4}{3} \Lambda_{\mathrm{cc}}\right)
$$

We observe an interesting fact, that all dependence on the form factor in (A7) is only via shifted one $\mathcal{F}_{2}$. The variation of the nonlocal Weyl square operator on a MSS background can be written also in a manifestly self-adjoint form in $D=4$, namely

$$
\begin{aligned}
& \frac{1}{2} \delta^{2}\left(\int d^{4} x \sqrt{|g|} C_{\mu \nu \rho \sigma} \mathcal{F}(\square) C^{\mu \nu \rho \sigma}\right) \\
& \overline{\overline{\mathrm{MSS}}} \int d^{4} x \sqrt{|g|}\left[h_{\mu \nu}\left(\frac{4}{9} \Lambda_{\mathrm{cc}}^{2}-\Lambda_{\mathrm{cc}} \square+\frac{1}{2} \square^{2}\right) \mathcal{F}\left(\square+\frac{4}{3} \Lambda_{\mathrm{cc}}\right) h_{\mu \nu}\right. \\
& \quad+h\left[\left(-\frac{1}{9} \Lambda_{\mathrm{cc}}^{2}+\frac{1}{4} \Lambda_{\mathrm{cc}} \square-\frac{1}{8} \square^{2}\right) \mathcal{F}\left(\square+\frac{4}{3} \Lambda_{\mathrm{cc}}\right)-\left(\frac{1}{12} \Lambda_{\mathrm{cc}} \square+\frac{1}{24} \square^{2}\right) \mathcal{F}\left(\square+4 \Lambda_{\mathrm{cc}}\right)\right] h \\
& \quad+h_{\mu \nu} \nabla_{\mu} \nabla_{\nu}\left(\frac{1}{3} \Lambda_{\mathrm{cc}}+\frac{1}{6} \square\right) \mathcal{F}\left(\square+4 \Lambda_{\mathrm{cc}}\right) h+h\left(\frac{1}{3} \Lambda_{\mathrm{cc}}+\frac{1}{6} \square\right) \mathcal{F}\left(\square+4 \Lambda_{\mathrm{cc}}\right) \nabla_{\mu} \nabla_{\nu} h_{\mu \nu} \\
& \left.\quad-h_{\mu \nu} \nabla_{\mu}\left(\frac{1}{3} \Lambda_{\mathrm{cc}}+\square\right) \mathcal{F}\left(\square+3 \Lambda_{\mathrm{cc}}\right) \nabla_{\rho} h_{\nu \rho}+\frac{1}{3} h_{\mu \nu} \nabla_{\mu} \nabla_{\nu} \mathcal{F}\left(\square+4 \Lambda_{\mathrm{cc}}\right) \nabla_{\rho} \nabla_{\sigma} h_{\rho \sigma}\right] .
\end{aligned}
$$

It can easily be seen that in this self-adjoint form we encounter three different shifts of the argument of the form factor by $4 / 3 \Lambda_{\mathrm{cc}}, 3 \Lambda_{\mathrm{cc}}$, and $4 \Lambda_{\mathrm{cc}}$, respectively.

The above results for the second variations were checked using various methods. First, all expressions can be put in the self-adjoint form of the operator of the second order variational derivative. Second, all the variations, except for the term with a cosmological constant only, are invariant under the substitution $h_{\mu \nu} \rightarrow h_{\mu \nu}+\nabla_{(\mu} \xi_{\nu)}$ for all left or right instances of the fluctuations of metric, where $\xi_{\nu}$ is an arbitrary vector field and when the on-shell background is used. This is the statement of gauge invariance of the action with respect to general coordinate transformations. Last but not least, the second variations were checked against conformal invariance of the action with two Weyl tensors in $D=4$. More precisely, it had been checked that for metric fluctuations of the form $h_{\mu \nu}=\omega^{2}(x) g_{\mu \nu}$ the second variation of such an action on any MSS background vanishes.
[1] Y. V. Kuzmin, The convergent nonlocal gravitation. (in Russian), Yad. Fiz. 50, 1630 (1989) [Sov. J. Nucl. Phys. 50, 1011 (1989)].

[2] N. V. Krasnikov, Nonlocal gauge theories, Teor. Mat. Fiz. 73, 235 (1987) [Theor. Math. Phys. 73, 1184 (1987)].

[3] E. T. Tomboulis, Superrenormalizable gauge and gravitational theories, arXiv:hep-th/9702146v1; Renormalization and unitarity in higher derivative and nonlocal gravity theories, Mod. Phys. Lett. A 30, 1540005 (2015).

[4] J. Khoury, Fading gravity and self-inflation, Phys. Rev. D 76, 123513 (2007).

[5] L. Modesto, Super-renormalizable quantum gravity, Phys. Rev. D 86, 044005 (2012); Super-renormalizable multidimensional quantum gravity, Astron. Rev. 8, 4 (2013); Multidimensional finite quantum gravity, arXiv: 1402.6795 .

[6] L. Modesto and L. Rachwal, Super-renormalizable and finite gravitational theories, Nucl. Phys. B889, 228 (2014).
[7] F. Briscese, L. Modesto, and S. Tsujikawa, Superrenormalizable or finite completion of the Starobinsky theory, Phys. Rev. D 89, 024029 (2014).

[8] G. Calcagni and L. Modesto, Nonlocal quantum gravity and M-theory, Phys. Rev. D 91, 124059 (2015).

[9] S. Giaccari and L. Modesto, Nonlocal supergravity, Phys. Rev. D 96, 066021 (2017).

[10] S. Alexander, A. Marciano, and L. Modesto, The hidden quantum groups symmetry of super-renormalizable gravity, Phys. Rev. D 85, 124030 (2012).

[11] F. Briscese, A. Marciano, L. Modesto, and E. N. Saridakis, Inflation in (super-)renormalizable gravity, Phys. Rev. D 87, 083507 (2013).

[12] G. V. Efimov, Nonlocal Interactions [in Russian] (Nauka, Moscow, 1977); V. A. Alebastrov and G. V. Efimov, A proof of the unitarity of S-matrix in a nonlocal quantum field theory, Commun. Math. Phys. 31, 1 (1973); Causality in the quantum field theory with the nonlocal interaction, Commun. Math. Phys. 38, 11 (1974); G. V. Efimov, Amplitudes in nonlocal theories at high energies, 
Teor. Mat. Fiz. 128, 395 (2001) [Theor. Math. Phys. 128, 1169 (2001)].

[13] S. Giaccari, L. Modesto, L. Rachwal, and Y. Zhu, Finite entanglement entropy of black holes, Eur. Phys. J. C 78, 459 (2018).

[14] P. Donà, S. Giaccari, L. Modesto, L. Rachwal, and Y. Zhu, Scattering amplitudes in super-renormalizable gravity, J. High Energy Phys. 08 (2015) 038.

[15] R. Pius and A. Sen, Cutkosky rules for superstring field theory, J. High Energy Phys. 10 (2016) 024.

[16] A. Sen, Unitarity of superstring field theory, J. High Energy Phys. 12 (2016) 115.

[17] P. Chin and E. T. Tomboulis, Nonlocal vertices and analyticity: Landau equations and general Cutkosky rule, J. High Energy Phys. 06 (2018) 014.

[18] F. Briscese and L. Modesto, Cutkosky rules and perturbative unitarity in Euclidean nonlocal quantum field theories, arXiv:1803.08827.

[19] L. Modesto, J. W. Moffat, and P. Nicolini, Black holes in an ultraviolet complete quantum gravity, Phys. Lett. B 695, 397 (2011).

[20] V. P. Frolov and G. A. Vilkovisky, Quantum gravity removes classical singularities and shortens the life of black holes, INIS Report No. IC-79-69, 1979.

[21] V. P. Frolov and G. A. Vilkovisky, Spherically symmetric collapse in quantum gravity, Phys. Lett. B 106, 307 (1981).

[22] V. P. Frolov, Information loss problem and a "black hole" model with a closed apparent horizon, J. High Energy Phys. 05 (2014) 049.

[23] V. P. Frolov, Do black holes exist?, arXiv:1411.6981.

[24] V. P. Frolov, A. Zelnikov, and T. de Paula Netto, Spherical collapse of small masses in the ghost-free gravity, J. High Energy Phys. 06 (2015) 107.

[25] V. P. Frolov, Mass-Gap for Black Hole Formation in Higher Derivative and Ghost Free Gravity, Phys. Rev. Lett. 115, 051102 (2015).

[26] V. P. Frolov, Notes on non-singular models of black holes, Phys. Rev. D 94, 104056 (2016).

[27] V. P. Frolov and A. Zelnikov, Radiation from an emitter in the ghost free scalar theory, Phys. Rev. D 93, 105048 (2016).

[28] C. Bambi, D. Malafarina, and L. Modesto, Non-singular quantum-inspired gravitational collapse, Phys. Rev. D 88, 044009 (2013).

[29] C. Bambi, D. Malafarina, and L. Modesto, Terminating black holes in asymptotically free quantum gravity, Eur. Phys. J. C 74, 2767 (2014).

[30] G. Calcagni, L. Modesto, and P. Nicolini, Super-accelerating bouncing cosmology in asymptotically-free nonlocal gravity, Eur. Phys. J. C 74, 2999 (2014).

[31] A. S. Koshelev, L. Modesto, L. Rachwal, and A. A. Starobinsky, Occurrence of exact $R^{2}$ inflation in nonlocal UV-complete gravity, J. High Energy Phys. 11 (2016) 067.

[32] Y. D. Li, L. Modesto, and L. Rachwal, Exact solutions and spacetime singularities in nonlocal gravity, J. High Energy Phys. 12 (2015) 173.

[33] G. Calcagni, M. Montobbio, and G. Nardelli, Localization of nonlocal theories, Phys. Lett. B 662, 285 (2008); G. Calcagni and G. Nardelli, Nonlocal gravity and the diffusion equation, Phys. Rev. D 82, 123518 (2010).
[34] Y.S. Myung, Entropy of a black hole in infinite-derivative gravity, Phys. Rev. D 95, 106003 (2017).

[35] M. Asorey, J. L. Lopez, and I. L. Shapiro, Some remarks on high derivative quantum gravity, Int. J. Mod. Phys. A 12, 5711 (1997).

[36] I. L. Shapiro, Counting ghosts in the ghost-free nonlocal gravity, Phys. Lett. B 744, 67 (2015).

[37] L. Modesto and I. L. Shapiro, Superrenormalizable quantum gravity with complex ghosts, Phys. Lett. B 755, 279 (2016).

[38] L. Modesto, Super-renormalizable or finite Lee-Wick quantum gravity, Nucl. Phys. B909, 584 (2016).

[39] R. E. Cutkosky, P. V. Landshoff, D. I. Olive, and J. C. Polkinghorne, A non-analytic S matrix, Nucl. Phys. B12, 281 (1969).

[40] F. d. O. Salles and I. L. Shapiro, Do we have unitary and (super)renormalizable quantum gravity below the Planck scale?, Phys. Rev. D 89, 084054 (2014); Erratum 90, 129903 (2014).

[41] L. Modesto and L. Rachwal, Nonlocal quantum gravity: A review, Int. J. Mod. Phys. D 26, 1730020 (2017).

[42] A. S. Koshelev, Stable analytic bounce in nonlocal EinsteinGauss-Bonnet cosmology, Classical Quantum Gravity 30, 155001 (2013).

[43] T. Biswas, A. Conroy, A. S. Koshelev, and A. Mazumdar, Generalized ghost-free quadratic curvature gravity, Classical Quantum Gravity 31, 015022 (2014); Erratum 31, 159501 (2014).

[44] A. S. Koshelev, K. Sravan Kumar, and A. A. Starobinsky, $R^{2}$ inflation to probe non-perturbative quantum gravity, J. High Energy Phys. 03 (2018) 071.

[45] L. Modesto, M. Piva, and L. Rachwal, Finite quantum gauge theories, Phys. Rev. D 94, 025021 (2016).

[46] A. Accioly, A. Azeredo, and H. Mukai, Propagator, tree-level unitarity and effective nonrelativistic potential for higher-derivative gravity theories in D dimensions, J. Math. Phys. 43, 473 (2002).

[47] K. S. Stelle, Renormalization of higher derivative quantum gravity, Phys. Rev. D 16, 953 (1977).

[48] I. L. Buchbinder, S. D. Odintsov, and I. L. Shapiro, Effective action in quantum gravity (IOP Publishing, Bristol, 1992).

[49] P. Van Nieuwenhuizen, On ghost-free tensor Lagrangians and linearized gravitation, Nucl. Phys. B60, 478 (1973).

[50] G. Calcagni and L. Modesto, Stability of Schwarzschild singularity in nonlocal gravity, Phys. Lett. B 773, 596 (2017).

[51] G. Calcagni, L. Modesto, and Y.S. Myung, Black-hole formation and stability in nonlocal gravity, Phys. Lett. B 783, 19 (2018).

[52] M. Eran, Higher-derivative gauge and gravitational theories (supersymmetry), Ph.D. thesis, Calif. U. Los Angeles, 1998.

[53] L. Modesto and L. Rachwal, Universally finite gravitational and gauge theories, Nucl. Phys. B900, 147 (2015).

[54] L. Modesto and L. Rachwal, Finite conformal quantum gravity and nonsingular spacetimes, arXiv:1605.04173.

[55] C. Bambi, L. Modesto, and L. Rachwal, Spacetime completeness of non-singular black holes in conformal gravity, J. Cosmol. Astropart. Phys. 05 (2017) 003. 
[56] C. Bambi, L. Modesto, S. Porey, and L. Rachwal, Black hole evaporation in conformal gravity, J. Cosmol. Astropart. Phys. 09 (2017) 033.

[57] Y. S. Myung, Renormalizability and Newtonian potential in scale-invariant gravity, arXiv:1708.03451.

[58] B. Allen, The graviton propagator in de Sitter space, Phys. Rev. D 34, 3670 (1986).

[59] T. Biswas, A. S. Koshelev, and A. Mazumdar, Gravitational theories with stable (anti-)de Sitter backgrounds, Fundam. Theor. Phys. 183, 97 (2016).

[60] T. Biswas, A. S. Koshelev, and A. Mazumdar, Consistent higher derivative gravitational theories with stable de Sitter and anti-de Sitter backgrounds, Phys. Rev. D 95, 043533 (2017).

[61] L. Modesto, L. Rachwal, and I. L. Shapiro, Renormalization group in super-renormalizable quantum gravity, Eur. Phys. J. C 78, 555 (2018).

[62] G. Calcagni, L. Modesto, and G. Nardelli, Nonperturbative spectrum of nonlocal gravity, arXiv:1803.07848.

[63] G. Calcagni, L. Modesto, and G. Nardelli, Initial conditions and degrees of freedom of nonlocal gravity, J. High Energy Phys. 05 (2018) 087.
[64] A. S. Koshelev, Nonlocal SFT tachyon and cosmology, J. High Energy Phys. 04 (2007) 029.

[65] I. Y. Aref'eva and I. V. Volovich, Quantization of the Riemann zeta-function and cosmology, Int. J. Geom. Methods Mod. Phys. 04, 881 (2007); A. S. Koshelev and S. Y. Vernov, Analysis of scalar perturbations in cosmological models with a nonlocal scalar field, Classical Quantum Gravity 28, 085019 (2011).

[66] R. P. Woodard, Nonlocal models of cosmic acceleration, Found. Phys. 44, 213 (2014).

[67] M. Ostrogradsky, Mémoires sur les équations différentielles, relatives au problème des isopérimètres (In French), Mem. Acad. St. Petersbourg 6, 385 (1850).

[68] V. Balasubramanian, J. de Boer, and D. Minic, Notes on de Sitter space and holography, Classical Quantum Gravity 19, 5655 (2002); Exploring de Sitter space and holography, Ann. Phys. (Amsterdam) 303, 59 (2003).

[69] L. Rachwal, Models for RG running for gravitational couplings and applications, Ph.D. thesis, SISSA, 2013, http://inspirehep .net/record/1323358/files/Thesis_Rachwal.pdf. 